Algebraic 85 Geometric Topology

Volume 2 (2002) 1001-1050

Published: 5 November 2002

ATG

\title{
The Configuration space integral for links in $\mathbb{R}^{3}$
}

\author{
SylVAIN POIRIER
}

\begin{abstract}
The perturbative expression of Chern-Simons theory for links in Euclidean 3-space is a linear combination of integrals on configuration spaces. This has successively been studied by Guadagnini, Martellini and Mintchev, Bar-Natan, Kontsevich, Bott and Taubes, D. Thurston, Altschuler and Freidel, Yang and others. We give a self-contained version of this study with a new choice of compactification, and we formulate a rationality result.
\end{abstract}

AMS Classification $57 \mathrm{M} 25 ; 57 \mathrm{~J} 28$

Keywords Feynman diagrams, Vassiliev invariants, configuration space, compactification

\section{Introduction}

The perturbative expression of Chern-Simons theory for links in Euclidean 3space in the Landau gauge with the generic (universal) gauge group, that we call "configuration space integral" (according to a suggestion of D.Bar-Natan), is a linear combination of integrals on configuration spaces. It has successively been studied by E. Guadagnini, M. Martellini and M. Mintchev [GMM], D.BarNatan [BN2], M. Kontsevich [Ko], Bott and Taubes [BT], Dylan Thurston [Th] (who explained in details the notion of degree of a map), Altschuler and Freidel $[\mathrm{AF}]$, Yang $[\mathrm{Ya}]$ and others. We give a self-contained version of this study with a new choice of compactification, and formulate a rationality result.

\subsection{Definitions}

Let $M$ be a compact one-dimensional manifold with boundary. Let $L$ denote an imbedding of $M$ into $\mathbb{R}^{3}$. We say that $L$ is a link if we moreover have the condition that the boundary of $M$ is empty. The most important results in this article only concern the case of links, but we allow the general case in our definitions in order to prepare the next article.

And a link $L$ it is a knot when $M=S^{1}$. 
A diagram with support $M$, is the data of :

(1) A graph made of a finite set $V=U \cup T$ of vertices and a set $E$ of edges which are pairs of elements of $V$, such that : the elements of $U$ are univalent (belong to precisely one edge) and the elements of $T$ are trivalent, and every connected component of this graph meets $U$.

(2) An isotopy class $\sigma$ of injections $i$ of $U$ into the interior of $M$.

This definition of a diagram differs from the usual one in that it excludes the double edges ( $\bigcirc-$ ) and the loops (o-). This will be justified by the fact that the integral that we shall define makes no sense on loops, and by Proposition 1.8 which will allow us to exclude diagrams with a double edge, among others.

For a diagram $\Gamma$, the degree of $\Gamma$ is the number

$$
\operatorname{deg} \Gamma=\frac{1}{2} \# V=\# E-\# T=\frac{1}{3}(\# E+\# U) .
$$

An isomorphism between two diagrams $\Gamma=(V, E, \sigma)$ and $\Gamma^{\prime}=\left(V^{\prime}, E^{\prime}, \sigma^{\prime}\right)$, is a bijection from $V$ to $V^{\prime}$ which transports $E$ to $E^{\prime}$ and $\sigma$ to $\sigma^{\prime}$.

Let $|\Gamma|$ denote the number of automorphisms of $\Gamma$.

Let $\mathcal{D}(M)$ denote the set of diagrams with support $M$ up to isomorphism, and $\mathcal{D}_{n}(M)$ its subset made of the degree $n$ diagrams, for all $n \in \mathbb{N}$.

$\mathcal{D}_{0}(M)$ has one element which is the empty diagram.

$\mathcal{D}_{1}\left(S^{1}\right)$ also has only one element, which will be denoted by $\theta$, for it looks like the letter $\theta$.

An orientation $o$ of a diagram $\Gamma$ is the data of an orientation of all its vertices, where the orientation of a trivalent vertex is a cyclic order on the set of the three edges it belongs to, and the orientation of a univalent vertex is a local orientation of $M$ near its image by $i$.

Let $\mathcal{A}_{n}(M)$ denote the real vector space generated by the set of oriented diagrams of degree $n$, and quotiented by the following AS, IHX and STU relations. The image of an oriented diagram $\Gamma$ in the space $\mathcal{A}_{n}$ is denoted by $[\Gamma]$.

The AS relation says that if $\Gamma$ and $\Gamma^{\prime}$ differ by the orientation of exactly one vertex, then $\left[\Gamma^{\prime}\right]=-[\Gamma]$.

The IHX and STU relations are respectively defined by the following formulas.

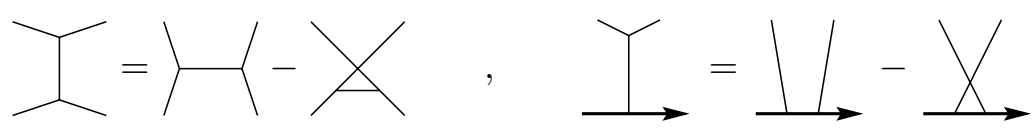

Algebraic 83 Geometric Topology, Volume 2 (2002) 
These formulas relate diagrams which are identical outside one place, where they differ according to the figures. By convention for the figures, the orientation at each trivalent vertex is the given counterclockwise order of the edges, and the orientation of each univalent vertex is given by the drawn orientation of the bold line (which represents $M$ locally).

Now let $\mathcal{A}(M)$ denote the space

$$
\mathcal{A}(M)=\prod_{n \in \mathbb{N}} \mathcal{A}_{n}(M)
$$

A configuration of a diagram $\Gamma \in \mathcal{D}(M)$ on $L$ is a map from the set $V$ of vertices of $\Gamma$ to $\mathbb{R}^{3}$, which is injective on each edge, and which coincides on $U$ with $L \circ i$ for some $i$ in the class $\sigma$. The configuration space $C_{\Gamma}(L)$ (or simply $C_{\Gamma}$ if $L$ is fixed) of a diagram $\Gamma \in \mathcal{D}(M)$ on $L$ is the set of these configurations.

$C_{\Gamma}$ has a natural structure of a smooth manifold of dimension $\# U+3 \# T=$ $2 \# E$. We shall canonically associate an orientation of $C_{\Gamma}$ to the data of an orientation of $\Gamma$ and an orientation of all edges of $\Gamma$, such that it is reversed when we change the orientation of one vertex or one edge (Subsection 4.2).

When orientations of all edges are chosen, we have the following canonical map $\Psi$ from $C_{\Gamma}$ to $\left(S^{2}\right)^{E}$ : for a configuration $x \in C_{\Gamma}$ and $e=\left(v, v^{\prime}\right), \Psi(x)_{e}$, is the direction of $x\left(v^{\prime}\right)-x(v)$.

Let $\omega$ denote the standard volume form on $S^{2}$ with total mass 1, and let

$$
\Omega=\bigwedge^{E} \omega
$$

denote the corresponding volume form on $\left(S^{2}\right)^{E}$.

Now the configuration space integral is $Z(L) \in \mathcal{A}(M)$ :

$$
Z(L)=\sum_{\Gamma \in \mathcal{D}(M)} \frac{I_{L}(\Gamma)}{|\Gamma|}[\Gamma]
$$

where

$$
I_{L}(\Gamma)=\int_{C_{\Gamma}(L)} \Psi^{*} \Omega
$$

We shall see that this integral $I_{L}(\Gamma)$ converges. The sign of the product $I_{L}(\Gamma)[\Gamma]$ will not depend on choices of orientations. Then $Z(L)$ also converges because it is a finite sum in each $\mathcal{A}_{n}(M)$.

By convention, the zero-degree part of $Z(L)$ is $1=[\emptyset]$.

As an introduction to the study of $Z(L)$, let us first recall the properties of its degree one part when $L$ is a link. 
There are two types of degree one diagrams: the chords $c_{m, m^{\prime}}$ between two different components $m$ and $m^{\prime}$ of $M$, and the chords $\theta_{m}$ with both ends on the same component $m$ of $M$. There are no IHX or STU relations between them, so the degree one part of $Z$ consists of the data of the integrals $I_{L}\left(c_{m, m^{\prime}}\right)$ and $I_{L}\left(\theta_{m}\right)$.

The space $C_{c_{m, m^{\prime}}}(L)$ is identical to $m \times m^{\prime}$, so, since $M$ has no boundary, it is diffeomorphic to the torus $S^{1} \times S^{1}$. Now, $\Psi$ is a smooth map from $m \times m^{\prime}$ to $S^{2}$, so the integral $I_{L}\left(c_{m, m^{\prime}}\right)=\int_{c_{m, m^{\prime}}} \Psi^{*} \omega$ is equal to the degree of the map $\Psi$ : it takes values in $\mathbb{Z}$. Precisely, it is equal to the linking number of $L(m)$ and $L\left(m^{\prime}\right)$.

The space $C_{\theta}$ is diffeomorphic to the noncompact surface $\left.S^{1} \times\right] 0,1[$. So the integral $I_{L}(\theta)$ is not the degree of a map. In fact it is known as the Gauss integral, usually considered in the case of a knot. It takes all real values on each isotopy class of knots. In the case of an almost planar knot, it approaches an integer which is the writhe or self-linking number of this knot (the number of crossings counted with signs).

In order to express some theorems about this expression $Z(L)$, we need first to recall some usual algebraic structures.

\subsection{Algebraic structures on the spaces $\mathcal{A}$ of diagrams}

For disjoint $M$ and $M^{\prime}$ included in some $M^{\prime \prime}$ such that their interiors do not meet, one can consider the bilinear map from $\mathcal{A}(M) \times \mathcal{A}\left(M^{\prime}\right)$ to $\mathcal{A}\left(M^{\prime \prime}\right)$ defined by: for every oriented diagrams $\Gamma$ with support $M$ and $\Gamma^{\prime}$ with support $M^{\prime}$, $[\Gamma] \cdot\left[\Gamma^{\prime}\right]=\left[\Gamma \sqcup \Gamma^{\prime}\right]$. This bilinear map is graded, that is, it sends $\mathcal{A}_{n}(M) \times \mathcal{A}_{n^{\prime}}\left(M^{\prime}\right)$ to $\mathcal{A}_{n+n^{\prime}}\left(M^{\prime \prime}\right)$.

Now, let $J=[0,1]$. It is well-known [BN1] that the bilinear product on $\mathcal{A}(J)$ defined by gluing the two copies of $J$ preserving the orientation, defines a commutative algebra structure with unit $1=[\emptyset]$, and that $\mathcal{A}(J)$ can be identified with $\mathcal{A}\left(S^{1}\right)$.

Similarly, for every $M$ and every connected component $m$ of $M$ with an orientation (either with boundary or not), the insertion of $J$ at some point of $m$ preserving the orientation provides an $\mathcal{A}(J)$-module structure on $\mathcal{A}(M)$ : it is well-known [BN1] that the result does not depend on the place of the insertion, modulo the AS, IHX and STU relations. 


\subsection{Results on the configuration space integral}

The convergence of the integrals $I_{L}(\Gamma)$ comes from the following proposition which will be proved in Section 2 .

Proposition 1.1 There exists a compactification of $C_{\Gamma}$ into a manifold with corners $\overline{C_{\Gamma}}$, on which the canonical map $\Psi$ extends smoothly.

Bott and Taubes $[\mathrm{BT}]$ first proved the convergence of the integrals by compactifying the configuration space $C_{\Gamma}$ into a manifold with corners, where $\Psi^{*} \Omega$ extends smoothly. (Their compactification was a little stronger than ours).

The notion of a manifold with corners is defined in their Appendix; briefly, it means every point has a neighbourhood diffeomorphic to $]-1,1\left[{ }^{p} \times\left[0,1\left[^{n-p}\right.\right.\right.$ for some $p$ from 0 to $n$.

They restricted their interest to the case of knots, and they studied the variations of these integrals during isotopies, in order to check the invariance. So, they applied the Stokes theorem to express the variations of $I_{L}(\Gamma)$, in terms of integrals on the fibered spaces over the time, made of the faces of the $\overline{C_{\Gamma}}$. They found equalities between the integrals at certain faces of different $\overline{C_{\Gamma}}$, where the diagrams $\Gamma$ are the terms of an IHX or STU relation, and proved cancellation on the other faces except those of a special kind called "anomalous", which follow in some way the tangent map of $L$. The global effect of the contributions of the anomalous faces to the variations of the integral can be expressed by a universal constant $\alpha \in \mathcal{A}$, the so-called anomaly.

Using many of their arguments, we shall prove the following proposition for links (when $\partial M=\emptyset)$ :

Proposition 1.2 The variations of $Z(L)$ on each isotopy class of links are expressed by the formula

$$
Z(L)=Z^{0}(L) \prod_{m} \exp \left(I_{L}\left(\theta_{m}\right) \alpha^{(m)}\right)
$$

where $m$ runs over the connected components of $M$, and :

$Z^{0}(L) \in \mathcal{A}(M)$ is an isotopy invariant of $L$,

$\alpha$ is a universal constant in $\mathcal{A}(J)$ called the anomaly,

$\alpha^{(m)}$ means $\alpha$ acting on $\mathcal{A}(M)$ on the component $m$. 
(But if the boundary of $M$ was not empty, it would produce another boundary of the $\overline{C_{\Gamma}}$ and thus uncontrollable variations of $Z$.)

Altschuler and Freidel $[\mathrm{AF}]$ have written this formula in the case of knots. They and Dylan Thurston [Th] also prove that

Proposition 1.3 $Z^{0}$ is a universal Vassiliev invariant.

The degree one part of $\alpha$ is $\frac{[\theta]}{2}$, and it is not difficult to see that all even degree parts of $\alpha$ cancel for symmetry reasons (Lemma 6.5).

We shall also prove in Section 7 that

Proposition 1.4 The degree 3 part of the anomaly vanishes.

But first we shall prove the following Proposition 1.8 in Section 3:

Notation 1.5 Let $A$ be any nonempty subset of $V=U \cup T$. We denote

$$
\begin{aligned}
& E_{A}=\{e \in E \mid e \subseteq A\} \\
& E_{A}^{\prime}=\{e \in E \mid \#(e \cap A)=1\} .
\end{aligned}
$$

The cardinalities of these sets are related by the following formula which expresses the two ways of counting the half-edges of the vertices in $A$ :

$$
2 \# E_{A}+\# E_{A}^{\prime}=3 \#(A \cap T)+\#(A \cap U)
$$

Definition 1.7 A diagram $\Gamma$ is said to be principal if for any $A \subseteq T$ such that $\# A>1$ we have $\# E_{A}^{\prime} \geq 4$.

A diagram $\Gamma$ is said to be subprincipal if it obeys the two following conditions:

(1) For any $A \subseteq T$ such that $\# A>1$, we have $\# E_{A}^{\prime} \geq 3$

(2) For any two $A, A^{\prime} \subseteq T$ such that $\# A>1, \# A^{\prime}>1$ and $\# E_{A}^{\prime}=\# E_{A^{\prime}}^{\prime}=3$, we have $A \cap A^{\prime} \neq \emptyset$.

The interest of these definitions comes from the following properties:

Proposition 1.8 For a given $\Gamma$, there exists a $L$ such that $\operatorname{codim} \Psi\left(C_{\Gamma}\right)=0$ if and only if $\Gamma$ is principal. And the property that $\Gamma$ is subprincipal is a necessary condition so that codim $\Psi\left(C_{\Gamma}\right) \leq 1$. 
Remark 1.9 The equivalence is also probably true for the second case, when $L$ is generic. But we shall not use this result in this article. In fact, as what we only need is a necessary condition so that $\operatorname{codim} \Psi\left(C_{\Gamma}\right) \leq 1$, we shall not make use of Condition (2) in the definition of a subprincipal diagram.

In fact, Condition (1) implies that any sets $A, A^{\prime} \subseteq T$ such that $\# A>1$, $\# A^{\prime}>1$ and $\# E_{A}^{\prime}=\# E_{A^{\prime}}^{\prime}=3$ are either disjoint or one is included in the other (this is a bit tedious to prove and we shall not need it).

The idea of the proof of Proposition 1.2 is that for every $n$, the degree $n$ part $Z_{n}^{0}$ of $Z^{0}$ can be seen as the degree of a certain map from a glued union of configuration spaces which are given rational multiples of the classes of their diagrams in $\mathcal{A}(M)$ as coefficients, to a certain manifold $\left(S^{2}\right)^{N}$ (where we can take for $N$ the maximum number of edges of degree $n$ diagrams).

This implies that $Z_{n}^{0}$ is a rational linear combination of the $[\Gamma]$ for the degree $n$ diagrams $\Gamma$. More precisely we have the following proposition, which will be proved together with Proposition 1.2 in Section 6.

Notation 1.10 For a diagram $\Gamma$, let $u_{\Gamma}$ be the number of univalent vertices of $\Gamma$, and $e_{\Gamma}$ be its number of edges (we have $u_{\Gamma}+e_{\Gamma}=3 \operatorname{deg} \Gamma$ ).

Let $k \in \mathbb{Z}, k \leq 2 n$, and let $\mathcal{A}_{n}^{k}(M)$ be the quotient space of $\mathcal{A}_{n}(M)$ by the subspace generated by the subprincipal diagrams $\Gamma$ such that $u_{\Gamma}=k-1$. (This implies, through the STU relation, $i$, the cancellation of all subprincipal diagrams with $u_{\Gamma}<k$.)

We must suppose that $k \leq 2 n$, else we would have $\mathcal{A}_{n}^{k}(M)=\{0\}$. Let $Z_{n}^{k}$ be the image of $Z_{n}$ in $\mathcal{A}_{n}^{k}(M)$. Proposition 1.8 implies that $\mathcal{A}_{n}^{2}(M)=\mathcal{A}_{n}(M)$, and that $\mathcal{A}_{n}^{3}(M)=\mathcal{A}_{n}(M)$ if $n>1$.

The reason for this construction of $\mathcal{A}_{n}^{k}(M)$ will appear in the proof of Lemma 5.6 which is used to obtain the following rationality result:

Proposition 1.11 We suppose $L$ is such that for all $m, I_{L}\left(\theta_{m}\right)$ is an integer. Let $n \geq 1, k \in \mathbb{Z}$ and $N=3 n-k$. Then $Z_{n}^{k}$ belongs to the integral lattice generated by the elements

$$
\frac{\left(N-e_{\Gamma}\right) !}{N ! 2^{e_{\Gamma}}}[\Gamma]
$$

in the space $\mathcal{A}_{n}^{k}(M)$, where $\Gamma$ runs over the degree $n$ principal diagrams. 
Remark 1.12 Here are two results which will be proved in a future article:

- The above proposition can be improved by saying that $Z_{n}$ belongs to the integral lattice generated by the elements

$$
\frac{[\Gamma]}{e_{\Gamma} ! 2^{e_{\Gamma}}}
$$

where $\Gamma$ runs over the set of principal diagrams.

- The degree 5 part of the anomaly cancels (this uses a Maple program).

The present rationality result can be compared to the one obtained for the Kontsevich integral: it was proved in [Le] that the degree $n$ part of the Kontsevich integral is a linear combination of chord diagrams (or of all diagrams, which is the same), where the coefficients are multiples of

$$
\frac{1}{(1 ! 2 ! \cdots n !)^{4}(n+1)} \text {. }
$$

For large values of $n$, these denominators are greater than ours, but have smaller prime factors.

I would like to express my thanks to Christine Lescop for the help during the preparation of this paper. I also thank Gregor Masbaum, Pierre Vogel, Alexis Marin, Su-Win Yang, Dylan Thurston, Dror Bar-Natan, Simon Willerton and Michael Polyak for their advice and comments.

\section{Compactification}

\subsection{The compactified space $\mathcal{H}(G)$ of a configuration space $C(G)$ of a graph}

Notation 2.1 If $A$ is a finite set with at least two elements, let $C^{A}$ denote the space of nonconstant maps from $A$ to $\mathbb{R}^{3}$ quotiented by the translationdilations group (that is the group of translations and positive homotheties of $\left.\mathbb{R}^{3}\right)$.

Note that the space $C^{A}$ is diffeomorphic to the sphere $S^{3 \# A-4}$ : choose an element $x \in A$ to be at the origin; then the images of the other elements of $A$ are to be considered modulo the dilations with center the origin. Thus it is compact. 
Notation 2.2 Let $G$ be a graph defined as a pair $G=(V, E)$ where $V$ is the set of "vertices" and $E$ is the set of "edges": $V$ is a finite set and $E$ is a set of pairs of elements of $V$. We suppose that $\# V \geq 2$ and that $G$ is connected.

We define the configuration space $C(G)$ as the space of maps from $V$ to $\mathbb{R}^{3}$ which map the two ends of every edge to two different points, modulo the dilations and translations (it is a dense open subset of $C^{V}$ ).

By an abuse of notation, any subset $A$ of $V$ will also mean the graph with the set $A$ of vertices and the set $E_{A}$ of edges (the set of edges $e \in E$ such that $e \subseteq A)$.

Let $R$ be the set of connected subsets $A$ of $V$ such that $\# A \geq 2$.

Let $\mathcal{H}(G)$ be the subset of $\prod_{A \in R} C^{A}$ made of the $x=\left(x_{A}\right)_{A \in R}$ such that $\forall A, B \in R, A \subseteq B \Rightarrow$ the restriction of $x_{B}$ to $A$ is either the constant map or the map $x_{A}$.

We define a canonical imbedding of $C(G)$ in $\mathcal{H}(G)$ by restricting any $f \in C(G)$ to each of the $A \in R$ : indeed, $\forall A \in R, \forall f \in C(G), f$ is not constant on $A$ because $A$ contains at least one edge, so the restriction of $f$ to $A$ is a welldefined element of $C^{A}$. Moreover, this is an imbedding because $V \in R$.

We can see that $\mathcal{H}(G)$ is compact, as a closed subset of the compact manifold $\prod_{A \in R} C^{A}$, for it is defined as an intersection of closed sets:

$\forall A, B \in R$ such that $A \subseteq B$, let us see why $\left\{\left(x_{A}, x_{B}\right) \in C^{A} \times C^{B} \mid\right.$ the restriction of $x_{B}$ to $A$ is either the constant map or the map $\left.x_{A}\right\}$ is closed, in the following way: by identifying each of $C^{A}, C^{B}$ as a sphere of representatives a convenient way (fixing one vertex in $A$ to the origin) in the respective linear space $\mathcal{L}, \mathcal{L}^{\prime}$ with the canonical linear projection $\pi$ from $\mathcal{L}^{\prime}$ onto $\mathcal{L}$, then the above set is the projection by $\left(x_{A}, x_{B}, \lambda\right) \mapsto\left(x_{A}, x_{B}\right)$ of the closed thus compact set of $\left(x_{A}, x_{B}, \lambda\right) \in C^{A} \times C^{B} \times[0,1]$ such that $\pi\left(x_{B}\right)=\lambda x_{A}$.

In the next subsection, we shall prove that $\mathcal{H}(G)$ is a manifold with corners, and that it is a compactification of $C(G)$. Now we are going to describe the strata of $\mathcal{H}(G)$. First, let us introduce this description intuitively:

An element $x$ of a stratum of $\mathcal{H}(G)$ will be a limit of elements of $C(G)$. This limit is first described by its projection to $C^{V}$; but some edges will collapse if $x$ is not in the interior stratum $C(G)$. Group the collapsing edges into connected components $A$, zoom in on each such $A$ and describe the relative positions of the vertices in $A$ : it is an element of $C^{A}$. If some edges in $A$ still collapse, do the same operations until all the relative positions of points in connected subdiagrams are described. The set of all sets $A$ on which you have zoomed in forms a subset $S$ of $R$. 
Notation 2.3 In all the following, $S$ will denote any subset of $R$ such that $V \in S$ and any two elements of $S$ are either disjoint or included one into the other. Let $S^{\prime}=S-\{V\}$.

For any $A \in R$, let $G / A$ denote the graph obtained by identifying the elements of $A$ into one vertex, and deleting the edges in $E_{A}$.

For any $A \in S$, let $A / S$ denote the graph $A$ quotiented by the greatest elements $A_{i}$ of $S$ strictly included in $A$ (they are disjoint and any element of $S$ strictly included in $A$ is contained in one of them).

Identify the space $C(A / S)$ to a subset of $C^{A}$ in the natural way (each $x \in$ $C(A / S)$ is then constant on each of the sets $\left.A_{i}\right)$ and let

$$
C(G, S)=\prod_{A \in S} C(A / S) \subseteq \prod_{A \in S} C^{A} .
$$

$\forall A \subseteq V, A \neq \emptyset$, let $\bar{A}$ denote the smallest element of $S$ containing $A$;

$\forall A \in S^{\prime}$, let $\widehat{A}$ denote the smallest element of $S$ that strictly contains $A$.

Let us first check that the last definitions make sense.

For any nonempty subset $A$ of $V$, the smallest element of $S$ containing $A$ is well-defined because first $A \subseteq V \in S$, then two elements of $S$ which contain $A$ cannot be disjoint, so one of them is included in the other.

The definition of $\widehat{A}$ is justified in the same way. This gives $S$ a tree structure.

Lemma 2.4 (1) For all $S$ and all $x \in C(G, S)$, there is a unique $\tilde{x} \in \mathcal{H}(G)$ which is an extension of $x$ to $R$. This defines an imbedding of $C(G, S)$ into $\mathcal{H}(G)$.

(2) This $\tilde{x}$ has the property that for all $A, B \in R$ with $A \subseteq B$, the restriction of $\tilde{x}_{B}$ to $A$ is constant if and only if $\bar{A} \neq \bar{B}$.

(3) For all $x \in \mathcal{H}(G)$, there is a unique $S$ such that the restriction of $x$ to $S$ belongs to $C(G, S)$.

Proof (1) First let us see the construction and uniqueness of $\tilde{x}$.

Since $\tilde{x}$ must belong to $\mathcal{H}(G)$, for all $A \in R$, the restriction of $\tilde{x}_{\bar{A}}=x_{\bar{A}}$ to $A$ must be either constant or equal to $\tilde{x}_{A}$. But it is not constant for the following reason: 
By definition of $\bar{A}, A$ is not reduced to one vertex in $\bar{A} / S$. Furthermore, since $A$ is connected, it has at least one common edge with $\bar{A} / S$. Then, since $x_{\bar{A}} \in C(\bar{A} / S)$, it cannot be constant on this edge.

Thus, for all $A \in R$, the value of $\tilde{x}_{A}$ is determined by $x_{\bar{A}}$. This proves the uniqueness of $\tilde{x}$.

As for the existence of $\tilde{x}$, the fact that this $\tilde{x}$ constructed above is actually an element of $\mathcal{H}(G)$, will be a consequence of (2).

Now, the fact that this defines an imbedding of $C(G, S)$ into $\mathcal{H}(G)$ is easy.

(2) $A \subseteq B$ implies $\bar{A} \subseteq \bar{B}$, so if $\bar{A} \neq \bar{B}$ then the restriction of $x_{\bar{B}}$ to $\bar{A}$ is constant, so the restriction of $\tilde{x}_{B}$ to $A$ is also constant.

If $\bar{A}=\bar{B}$, then $\tilde{x}_{A}$ and $\tilde{x}_{B}$ are both restrictions of $x_{\bar{A}}$, so $\tilde{x}_{A}$ is the restriction of $\tilde{x}_{B}$ to $A$.

(3) The existence and uniqueness of such an $S$ come from the following construction of $S$ as a function of an $x \in \mathcal{H}(G)$.

The elements of $S$ can be enumerated recursively, starting with its first element $A=V$. For any $A \in S$, consider the partition of $A$ defined by $x_{A}$ (i.e. the set of preimages of the singletons). Then, the greatest elements of $S$ strictly included in $A$ must be precisely the connected components, with cardinality greater than one, of the sets in this partition.

\subsection{Description of the corners of $\mathcal{H}(G)$}

Now we identify $C(G, S)$ with a subset of $\mathcal{H}(G)$ thanks to Lemma 2.4. A direct calculation shows that $\operatorname{dim} C(G, S)=\operatorname{dim} C(G)-\# S^{\prime}$. We are going to see the following

Proposition 2.5 $C(G, S)$ has a neighbourhood ${ }^{1}$ in $\mathcal{H}(G)$ which is diffeomorphic to a neighbourhood of $C(G, S) \times\{0\}^{S^{\prime}}$ in $C(G, S) \times\left[0,+\infty\left[{ }^{S^{\prime}}\right.\right.$. Thus, $\mathcal{H}(G)$ is a manifold with corners.

Intuitively, in the family of parameters $\left(u_{A}\right)_{A \in S^{\prime}}$, each $u_{A}$ will measure the relative size of $A$ and $\widehat{A}$.

\footnotetext{
${ }^{1}$ A neighbourhood of a subset $P$ of a topological space is a neighbourhood of every point of $P$; it does not necessarily contain the closure of $P$.
} 
This diffeomorphism is not completely canonical: to define it we have to choose for each $A \in S$ an identification of $C(A / S)$ with a smooth section of representatives in $\left(R_{A}\right)^{A / S}$ where $R_{A}$ is just a copy of $\mathbb{R}^{3}$ marked with the label $A$. For instance, suppose that $V$ is totally ordered and that for all $A \in R$ we fix the smallest vertex in $A$ at the origin of $R_{A}$; as for the dilations, just fix any choice of smooth normalisation.

Construction of a smooth map from a neighbourhood of $C(G, S) \times\{0\}^{S^{\prime}}$ in $C(G, S) \times\left[0,+\infty\left[{ }^{S^{\prime}}\right.\right.$ to a neighbourhood of $C(G, S)$ in $\mathcal{H}(G)$

Take a family $u=\left(u_{A}\right)_{A \in S^{\prime}}$ of positive real numbers and a family $f \in C(G, S)$, that is, $f=\left(f_{A}\right)_{A \in S}$ and $f_{A} \in R_{A}^{A / S}$. Then, define the net $\left(\phi_{A}\right)$ of correspondences from each space $R_{A}$ for $A \in S^{\prime}$ to the space $R_{\widehat{A}}$ by

$$
\begin{aligned}
\phi_{A}: R_{A} & \longrightarrow R_{\widehat{A}} \\
x & \longmapsto f_{\widehat{A}}(A)+u_{A} x .
\end{aligned}
$$

We construct an element $g$ of $\mathcal{H}(G)$ in the following way:

For all $B \in R$, we define the $C^{B}$ part of $g$ as a map from $B$ to $R_{\bar{B}}$ : first take for all $v \in B$ its image by $f_{\overline{\{v\}}}$ in $R_{\overline{\{v\}}}$, then compose all the maps $\phi_{A}$ above for the $A \in S$ such that $\overline{\{v\}} \subseteq A \subset \bar{B}$ to obtain an element of $R_{\bar{B}}$.

This map is not constant on $B$ for small enough values of $u$ because for $u=0$ it is equal to $f_{\bar{B}}$ which is not constant on $B$, so it gives an element of $C^{B}$.

This provides a well-defined smooth map from a neighbourhood $\mathcal{U}$ of $C(G, S) \times$ $\{0\}^{S^{\prime}}$ in $C(G, S) \times\left[0,+\infty\left[S^{S^{\prime}}\right.\right.$, to a subset of $\mathcal{H}(G)$, and its restriction to $C(G, S) \times\{0\}$ is the imbedding defined in Lemma 2.4.

It maps the interior of $\mathcal{U}$ to the stratum $C(G)$ of $\mathcal{H}(G)$, because when $\forall A \in S^{\prime}$, $u_{A}>0$, these operations are equivalent to first constructing the projection of $g$ in $C^{V}$, then restricting it to each element of $R$, because all the maps $\phi_{A}$ are dilations-translations of $\mathbb{R}^{3}$. So it maps $\mathcal{U}$ to $\mathcal{H}(G)$.

Remark 2.6 This diffeomorphism maps any $(f, u) \in \mathcal{U}$ into the stratum $C\left(G, S_{u}\right)$ where $S_{u}=\left\{A \in S \mid A=V\right.$ or $\left.u_{A}=0\right\}$.

\section{Proof that the map above has a smooth inverse}

We have to check that the $u_{A}\left(A \in S^{\prime}\right)$ and the $f_{A}(A \in S)$ can be expressed as smooth functions of a $g$ in a suitable neighbourhood of $C(G, S)$ in $\mathcal{H}(G)$. 
$f_{A} \in C(A / S)$ is the modification of $g_{A}$ obtained by mapping each $A^{\prime} \in S$ such that $\widehat{A^{\prime}}=A$ to $g_{A}\left(a^{\prime}\right)$ where $a^{\prime}$ is the smallest element of $A^{\prime}$. This $f_{A}$ maps the two ends of every edge of $A / S$ to two different points because it is in a suitable neighbourhood of $C(G, S)$ in $\mathcal{H}(G)$.

$u_{A}$ is the suitably defined size of $A$ in $g_{\widehat{A}}$.

The description of $\mathcal{H}(G)$ is now finished and we can proceed with the compactification of $C_{\Gamma}$.

\subsection{Construction of the compactified space $\overline{C_{\Gamma}}$ of $C_{\Gamma}$}

\section{Definition of $\overline{C_{\Gamma}}$}

Starting with a diagram $\Gamma \in \mathcal{D}(M)$, define the graph $G$ with the same set $V$ of vertices as $\Gamma$, and define the set of edges of $G$ to be the set of edges of $\Gamma$, plus all pairs of univalent vertices.

First, canonically imbed the space $C_{\Gamma}$ into the space

$$
\mathcal{H}(\Gamma)=\mathcal{H}(G) \times M^{U}
$$

and let the compactified space $\overline{C_{\Gamma}}$ of $C_{\Gamma}$ be its closure in $\mathcal{H}(\Gamma)$.

Now we are going to describe the corners of this space. For convenience, we suppose that $M$ is oriented and $\partial M=\emptyset$. The case of $\partial M \neq \emptyset$ will be seen at the end of this subsection.

The reason why we took all pairs of univalent vertices as edges of $G$ and not only the consecutive ones is to have $U$ connected even if $M$ is not, so that for any $\left(f, f^{\prime}\right) \in \overline{C_{\Gamma}} \subseteq \mathcal{H}(G) \times M^{U}$ such that $f^{\prime}$ is not the constant map to $M$, the restriction of the map $f_{\bar{U}}$ to $U$ is not constant and therefore is a picture of $f^{\prime}$. (For a knot, it makes no difference.)

\section{List of strata}

For commodity, let us fix an orientation on the manifold $M$.

A stratum of $C_{\Gamma}$ is labelled by the following data of $\left(S, P,(\leq)_{P}\right)$ :

- A set $S \subseteq R$ as above (Notation 2.3)

- A partition $P$ of $U$ (that will be the set of preimages of $f^{\prime}$ ), which satisfies one of the relations: 
(i) $P=\{U\}$

(ii) $P=$ the partition of $U$ induced by $\bar{U} / S$.

- A total order on each element of $P$.

such that: for any $A \in P$ and $B \in S, A \cap B$ with its total order is a set of consecutive elements for $\sigma$ respecting the orientation of $M$ (Thus, $A$ is mapped to a single connected component of $M$, and there is only one possibility for this total order if $A$ is not the whole preimage of a connected component of $M$ ).

\section{Description of strata}

The stratum with label $\left(S, P,(\leq)_{P}\right)$ is the set of elements $\left(f, f^{\prime}\right) \in \mathcal{H}(G) \times M^{U}$ such that:

- $f^{\prime}$ belongs to the closure of $\sigma$ and maps two univalent vertices to the same element of $M$ if and only if they belong to the same element of $P$.

- $f \in C(G, S)$

- Each ordered pair $(x, y)$ of elements of $U$ which belong to the same element $U_{1}$ of $P$ and are consecutive for $\leq_{U_{1}}$ is mapped by $f_{\{x, y\}}$ to the tangent vector to $L$ at their common image. Or equivalently, $\forall A \in S$, if $A \cap U$ is contained in one element $U_{1}$ of $P$, then $f_{A}$ maps $A \cap U$ to the straight line $l_{s}$ with direction the tangent vector $s$ to $L \circ f^{\prime}\left(U_{1}\right)$, preserving the order in the large sense.

- In case (ii), $f_{\bar{U}}$ must coincide on $U$ with $L \circ f^{\prime}$. Thus, we identify $R_{\bar{U}}$ to the image space $\mathbb{R}^{3}$ of $L$, because $L \circ f^{\prime}$ and $f_{\bar{U}}$ are not constant on $U$.

Note that in case (ii), the vertices in $V-\bar{U}$ are those which escape to infinity, or for which any path from them to a univalent vertex passes through trivalent vertices which escape to infinity (in case (i) there can be an undetermination).

We suppose that the univalent vertices are smaller so that they have priority to be at the origin of the spaces $R_{A}$.

\section{The neighbourhood of a stratum}

This is again a corner, with a family of independent positive parameters which are the same as before (indexed by $S^{\prime}$ ), plus one more in the case (i), which will be denoted by $u_{0}$.

To be quick, we shall only define the elements of $C_{\Gamma}$ which correspond to a family of strictly positive coordinates $\left(u_{A}\right)_{A \in S^{\prime}}$, and possibly $u_{0}$. 
This will be made in two steps: first an approximate definition, then a correction.

In the first step, we start with an element $\left(f, f^{\prime}\right)$ of the considered stratum with $f \in C(G, S)$, and a family $\left(u_{A}\right)$ of small enough strictly positive real numbers. The construction of Subsection 2.2 gives an identification of all the spaces $R_{A}$ together (because $u_{A}>0$ for all $A$ ), and thus an element of $C(G)$. There remains to identify one of the spaces (namely $R_{\bar{U}}$ ) to the image space $\mathbb{R}^{3}$ of $L$. In case (ii), this identification is already done; whereas in case (i), it will be

$$
\begin{aligned}
R_{\bar{U}} & \longrightarrow \mathbb{R}^{3} \\
x & \longmapsto L \circ f^{\prime}(U)+u_{0} x .
\end{aligned}
$$

In the second step, we have to choose for each $Q$ in the image of $L \circ f^{\prime}$ a diffeomorphism $\varphi_{Q}$ between two neighbourhoods of $Q$ which verifies the two conditions: first, it approximates the identity near $Q$ up to the first order; second, it maps the tangent line to the curve $L(M)$ at $Q$, to the curve $L(M)$ itself. This system of diffeomorphisms must depend smoothly on $f$.

Then for every $Q$, correct the map from $V$ to $\mathbb{R}^{3}$ of the first step by composing it with the map $\varphi_{Q}$ for the vertices in $f_{\bar{U}}{ }^{-1}(Q)$.

It can easily be seen that the resulting map extends as a smooth map on a neighbourhood of the stratum.

Case when $\partial M \neq \emptyset$

To the data $\left(S, P,(\leq)_{P}\right)$ we must add the datum of the set $f^{\prime}(U) \cap \partial M$. As for the parametrisation of the corners, each $x \in f^{\prime}(U) \cap \partial M$ gives the parameter $\operatorname{dist}\left(x, f_{1}^{\prime}(U)\right)$ for the $\left(f_{1}, f_{1}^{\prime}\right)$ in the neighbourhood of the stratum.

\section{End of proof of Proposition 1.1}

Now that we have compactified $C_{\Gamma}$ into a manifold with corners, we just have to check that $\Psi$ is smooth on $\overline{C_{\Gamma}}$. But $\Psi$ is just the map defined by the canonical projections of $\mathcal{H}(G)$ on the $C^{e} \approx S^{2}$ for $e \in E$, since $E \subseteq R$.

\subsection{List of faces}

The codimension 1 strata of $\overline{C_{\Gamma}}$ as constructed in the previous subsection can be classified into six types. In the first five types we have $f^{\prime}(U) \cap \partial M=\emptyset$.

(a) In case (i) there is the coordinate $u_{0}$, so $S$ must be reduced to $\{V\}$. 
In case (ii) with $S=\{V, A\}$, let us distinguish four types:

(b) $U \subseteq A$ (this corresponds to the case when some vertices go to infinity),

(c) $\# A=2, U \nsubseteq A$ and $E_{A}^{\prime} \neq \emptyset$; this is divided into two subtypes:

(c1) $A \subseteq T$ (thus $A \in E$ ),

(c2) $A \nsubseteq T$ (thus, either $A \subset U$ and $A \notin E$, or $A \nsubseteq U$ and $A \in E$ ).

(d) $\# A>2, U \nsubseteq A$ and $E_{A}^{\prime} \neq \emptyset$,

(a') $E_{A}^{\prime}=\emptyset$ (thus, $A \cap U \neq \emptyset$ and $U \nsubseteq A$ ).

Finally,

(e) Case (ii) with $S=\{V\}$ and $\#\left(f^{\prime}(U) \cap \partial M\right)=1$.

Definition 2.7 The faces of types (b), (c) and (d) will be called ordinary faces.

The faces of types (a) and (a') will be called anomalous faces ${ }^{2}$.

The faces of type (e) will be called extra faces; they do not exist in the case of links which is the main interest of this article.

We say that a face $F$ is degenerated if $\operatorname{codim} \Psi(F)>1$.

Notation 2.8 For $A \in R$ with $U \nsubseteq A$, let $F(\Gamma, A)$ denote the face of $\overline{C_{\Gamma}}$ defined by $S=\{V, A\}$.

In the next section we shall prove the following proposition about some types of faces which are degenerated. Its aim is to eliminate these diagrams for the next sections, as only the non-degenerated faces will need to be taken into account. For the same reason, we shall restrict the study to the configuration spaces of subprincipal diagrams, as the faces of other spaces are all degenerated thanks to Proposition 1.8.

Proposition 2.9 The only faces of $\overline{C_{\Gamma}}$ which can be non-degenerated are:

The anomalous faces in the connected case (type (a) faces with $\Gamma$ connected, and type (a') faces $F(\Gamma, A)$ with $A$ connected).

The faces of types (c) and (e).

The faces $F(\Gamma, A)$ of type (d) which satisfy the following conditions: each edge in $E_{A}^{\prime}$ meets at least two edges in $E_{A}$, and the number $\# E_{A}^{\prime}$ is 1 or 2 if $A \nsubseteq T$, and 3 or 4 if $A \subseteq T$.

\footnotetext{
${ }^{2}$ They are the "anomalous faces" of Bott and Taubes.
} 


\section{Proofs for the codimensions}

\subsection{Necessary conditions in Proposition 1.8}

Let $A \subseteq T, \# A>1$. Note that in the definition of principal or subprincipal diagrams, we can equivalently restrict the conditions to the sets $A$ which are connected: replacing a disconnected $A$ with one of its connected components does the trick.

Consider the following commutative diagram:

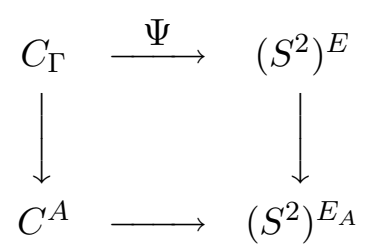

According to (1.6) we have $2 \# E_{A}+\# E_{A}^{\prime}=3 \# A$. Thus,

$$
\operatorname{dim} C^{A}=\operatorname{dim}\left(S^{2}\right)^{E_{A}}+\# E_{A}^{\prime}-4 .
$$

So, if $\Psi\left(C_{\Gamma}\right)$ has codimension 0 , then $\operatorname{dim} C^{A} \geq \operatorname{dim}\left(S^{2}\right)^{E_{A}}$ for all $A$, thus $\Gamma$ is principal.

This way, we can see that $\operatorname{codim} \Psi\left(C_{\Gamma}\right) \leq 1$ implies Condition (1) in the definition of a subprincipal diagram. Similarly, considering for any disjoint $A, A^{\prime} \subseteq T, \# A>1, \# A^{\prime}>1$, the natural map from $C_{\Gamma}$ to $\left(S^{2}\right)^{E_{A}} \times\left(S^{2}\right)^{E_{A^{\prime}}}$, we can see that it also implies Condition (2). Therefore, all diagrams such that $\operatorname{codim} \Psi\left(C_{\Gamma}\right) \leq 1$ are subprincipal.

\subsection{A general lemma on codimensions}

In the proofs of the propositions, we shall use several times the following argument to minorate the codimension of the images by $\Psi$ of certain subsets of $\mathcal{H}(G)$. It will especially apply to the properties of the image of a stratum of $\overline{C_{\Gamma}}$ in $C^{V} \times M^{U}$ by the canonical projection (values of $\left(f_{V}, f^{\prime}\right)$ ). It will play the role of a lemma but its expression is long and its proof trivial.

Let $\Gamma$ be a connected, subprincipal diagram.

Let $\mathcal{P} \subseteq R \backslash\{V\}$ be a set of pairwise disjoint sets of vertices.

Algebraic 83 Geometric Topology, Volume 2 (2002) 
Let $\Gamma^{\prime}=\Gamma / \mathcal{P}$ be the quotient diagram, made of

- a set $V^{\prime}=U^{\prime} \sqcup T^{\prime}$ of vertices such that:

(1) $V^{\prime}$ is the quotient of $V$ by the relation whose equivalent classes are the elements of $\mathcal{P}$ and singletons,

(2) $U^{\prime}$ is the image of $U$ by the canonical projection from $V$ to $V^{\prime}$.

- a set of edges $E^{\prime}=\left\{e \in E \mid \forall A \in \mathcal{P}, e \notin E_{A}\right\}$.

We can easily see that $\forall x \in U^{\prime},\left(x \in \mathcal{P}\right.$ or $\left(x \in U\right.$ and is univalent in $\left.\left.\Gamma^{\prime}\right)\right)$.

The same for $T^{\prime}: \forall x \in T^{\prime},\left(x \in \mathcal{P}\right.$ or $\left(x \in T\right.$ and is trivalent in $\left.\left.\Gamma^{\prime}\right)\right)$.

Let $U^{\prime \prime}=U^{\prime} \cap \mathcal{P}$ and $T^{\prime \prime}=T^{\prime} \cap \mathcal{P}$.

Let $L$ be a curve in $\mathbb{R}^{3}$, and let $C\left(\Gamma^{\prime}, L\right)$ be some configuration space of $\Gamma^{\prime}$ where every element of $U^{\prime}$ runs along $L$.

For any $x \in V^{\prime}$, let $n_{x}$ be the number of edges in $E^{\prime}$ which contain $x$. Then,

$$
\operatorname{dim}\left(S^{2}\right)^{E^{\prime}}-\operatorname{dim}\left(C\left(\Gamma^{\prime}, L\right)\right)=\sum_{x \in T^{\prime \prime}}\left(n_{x}-3\right)+\sum_{x \in U^{\prime \prime}}\left(n_{x}-1\right) .
$$

But (as $\Gamma$ is connected and subprincipal), we have $n_{x}-3 \geq 0$ for any $x \in T^{\prime \prime}$, and $n_{x}-1 \geq 0$ for any $x \in U^{\prime \prime}$, thus $\operatorname{dim}\left(S^{2}\right)^{E^{\prime}} \geq \operatorname{dim}\left(C\left(\Gamma^{\prime}, L\right)\right)$.

Therefore, the codimension $c$ of the image of the canonical map (restricted $\Psi$ ) from $C\left(\Gamma^{\prime}, L\right)$ to $\left(S^{2}\right)^{E^{\prime}}$ has the following properties:

(1) If $c=0$ then the group of translations or dilations letting $L$ invariant has dimension $0, n_{x}=3$ for every $x \in T^{\prime \prime}$ and $n_{x}=1$ for every $x \in U^{\prime \prime}$. Thus, as $\Gamma$ is subprincipal, every element of $U^{\prime \prime}$ contains at least two elements of $U$, and if $\Gamma$ is principal then $T^{\prime \prime}=\emptyset$.

(2) If $c=1$ then the above consequences of $c=0$ can suffer only one exception with the range of one unit.

In particular, if $L$ is contained in a straight line, then $c>1$.

\subsection{Proof of the sufficient condition in Proposition 1.8}

We shall prove now that if $\Gamma$ is principal then there exists a link $L$ such that $\operatorname{codim} \Psi\left(C_{\Gamma}\right)=0$. (This is just an elegant result which is not useful for the rest of the present article). 
So, we will study the existence of preimages for generic points in $\left(S^{2}\right)^{E}$.

For any $s=\left(s_{e}\right)_{e \in E} \in\left(S^{2}\right)^{E}$, let

$$
\mathcal{E}_{s}=\prod_{e \in E} \mathbb{R}^{3} / \mathbb{R} s_{e}
$$

Let $\Psi_{s}$ be the linear map from $\left(\mathbb{R}^{3}\right)^{V}$ to $\mathcal{E}_{s}$ defined by: for each edge $e=$ $\left(v, v^{\prime}\right) \in E \subseteq V^{2}$, and each $f \in\left(\mathbb{R}^{3}\right)^{V}, \Psi_{s}(f)_{e} \in \mathbb{R}^{3} / \mathbb{R} s_{e}$ is the image of $f\left(v^{\prime}\right)-f(v)$ by the canonical linear projection.

Now, the configuration space $C_{\Gamma}=C_{\Gamma}(L)$ of a link $L$ is a submanifold of $\left(\mathbb{R}^{3}\right)^{V}$ with the same dimension as $\mathcal{E}_{s}$, and we shall consider the restriction of $\Psi_{s}$ to it. Then, the set of preimages of 0 by $\Psi_{s}$ in $C_{\Gamma}$ corresponds bijectively to the disjoint union of sets of preimages by $\Psi$ in $C_{\Gamma}$ of all elements of the form $\left( \pm s_{e}\right)_{e \in E}$.

The condition "codim $\Psi\left(C_{\Gamma}\right)=0$ " can be reformulated in the form "There exists a configuration $x \in C_{\Gamma}$ at which the differential map $\mathrm{d} \Psi(x)$ is bijective". Let us analyse this differential map.

The tangent space of $C_{\Gamma}$ at $x$ is the topological closure $\overline{C_{\Gamma^{\prime}}}\left(L^{\prime}\right) \subseteq\left(\mathbb{R}^{3}\right)^{V}$ of the configuration space $C_{\Gamma^{\prime}}\left(L^{\prime}\right)$ where $L^{\prime}$ is the family of the tangent straight lines to the link $L$ at the positions of univalent vertices in the configuration $x$, and $\Gamma^{\prime}$ is obtained from $\Gamma$ by replacing its support with $U \times \mathbb{R}$.

Then, $\mathrm{d} \Psi(x)$ is the map $\Psi_{s}$ defined on $\overline{C_{\Gamma^{\prime}}}\left(L^{\prime}\right)$ where $s=\Psi(x)$.

What we have to prove is, for any principal diagram $\Gamma$, the existence of some $s, x$ and $L$ which verify these conditions. First, let us fix a family $L^{\prime}$ of pairwise disjoint straight lines indexed by $U$.

Lemma 3.1 For a generic $s \in\left(S^{2}\right)^{E}$, the map $\Psi_{s}$ is bijective from the affine space $\overline{C_{\Gamma^{\prime}}}\left(L^{\prime}\right)$ to $\mathcal{E}_{s}$.

Proof If it was not, as $\overline{C_{\Gamma^{\prime}}}\left(L^{\prime}\right)$ and $\mathcal{E}_{s}$ have the same dimension, then the kernel of the linear part of $\Psi_{s}$ would be nonzero, that is, there would be a nonzero element $x \in \overline{C_{\Gamma^{\prime}}}\left(T L^{\prime}\right)$ (where $T L^{\prime}$ is the family of parallel lines to the components of $L^{\prime}$ at the origin), in $\operatorname{Ker} \Psi_{s}$. But this is not possible for a generic $s$, thanks to Subsection 3.2, because $T L^{\prime}$ is invariant by dilations with center the origin.

Now, 0 has one preimage $x$ by $\Psi_{s}$ in $\overline{C_{\Gamma^{\prime}}}\left(L^{\prime}\right)$ for a generic $s$. This $x$ is not collapsed to one single point of $\mathbb{R}^{3}$ because the lines in $L^{\prime}$ are disjoint. Let us 
apply once more the result of Subsection 3.2: as we suppose that $\Gamma$ is principal, we find $T^{\prime \prime}=\emptyset$, and also $U^{\prime \prime}=\emptyset$ because all univalent vertices run over pairwise disjoint lines.

Therefore, we have $x \in C_{\Gamma^{\prime}}\left(L^{\prime}\right)$. Finally, we can find a link $L$ which is tangent to every line of $L^{\prime}$ at the position of every univalent vertex of $x$ respecting the datum $\sigma$ of $\Gamma$, so that at the same geometric configuration $x$, we have $\mathrm{d} \Psi(x) \approx$ $\Psi_{s}$ bijective from $T_{x} C_{\Gamma}=C_{\Gamma^{\prime}}\left(L^{\prime}\right)$ to $T_{s}\left(S^{2}\right)^{E} \approx \mathcal{E}_{s}$ where $s=\Psi(x)$.

\subsection{Proofs for the degenerated faces}

Remark 3.2 Suppose there exists a subset $A$ of $V$ such that $E_{A}^{\prime}=\emptyset$. Then consider the two subdiagrams $\Gamma_{1}$ and $\Gamma_{2}$ whose sets of vertices are $A$ and $V-A$ respectively. Then there is a canonical diffeomorphism from $C_{\Gamma}$ to an open subset of $C_{\Gamma_{1}} \times C_{\Gamma_{2}}$, which is delimited by a finite union of subspaces.

This gives a diffeomorphism between a type (a') face, and an open subset of the product of a type (a) face of $C_{\Gamma_{1}}$ with the space $C_{\Gamma_{2}}$. So it shows that the types (a) and (a') are fundamentally the same.

It also has the following consequence:

Lemma 3.3 A type (a) face with $\Gamma$ not connected, or a type (a') face with $A$ not connected, is degenerated.

Proof When $\Gamma$ is a disjoint union of two diagrams $\Gamma_{1}$ and $\Gamma_{2}$, the natural map from $\overline{C_{\Gamma}}$ to $\overline{C_{\Gamma_{1}}} \times \overline{C_{\Gamma_{2}}}$ maps a type (a) face of $C_{\Gamma}$ to a product of type (a) faces of $\bar{C}_{\Gamma_{1}}$ and $\bar{C}_{\Gamma_{2}}$, which has codimension 2 . Thus, as $\Psi$ is factorised through this, the image of its restriction to this face also has codimension at least two.

This concludes for the type (a), and the generalisation to (a') is immediate.

Lemma 3.4 The type (b) faces, and the type (d) faces $F(\Gamma, A)$ such that $\left(A \nsubseteq T\right.$ and $\left.\# E_{A}^{\prime}>2\right)$ or $\left(A \subseteq T\right.$ and $\left.\# E_{A}^{\prime}>4\right)$, are degenerated.

These are direct consequences of Subsection 3.2. For the type (b), the image of the univalent vertices is a vertex at least trivalent (because the diagram is subprincipal) which must stay at the origin, so that the codimension is at least 3. 
Lemma 3.5 Any type (d) face such that the vertex $v$ in $A$ of some edge in $E_{A}^{\prime}$ is either in $U$ or not bivalent in $A$, is degenerated.

Indeed, in such a face, each preimage of a point by $\Psi$ is at least one-dimensional because in the $C^{A}$ part of this preimage, this vertex $v$ can move freely in one direction while the others remain fixed. This direction is the tangent direction of $L$ at $f^{\prime}(v)$ if $v \in U$, or the one of its edge in $E_{A}$ if $v$ is univalent in $A$. This move is not eliminated by dilations because $\# A>2$.

This ends the proof of Proposition 2.9.

\section{Labelled diagrams and orientations}

\subsection{Labelled diagrams}

To formulate the degree $n$ part of $Z^{0}$ in terms of the degree of the map $\Psi$ from the glued configuration spaces to a product of spheres, we shall need to fix this product of spheres. Thus, all our edges will be labelled and oriented. We shall call that "labellings" of diagrams. We are going to reformulate the definition of the diagrams so that they will be intrinsically labelled.

Let us fix the degree $n$ of the diagrams. Their number of edges varies under STU but we must fix the number $N$ of their labels. So, we shall only work with the diagrams $\Gamma$ which have a number of edges $e_{\Gamma} \leq N$, and thus a number of univalent vertices $u_{\Gamma} \geq k=3 n-N$, and there may be some unused labels which will be called "absent edges".

Notation 4.1 If $A$ is a set of sets, let $\cup A$ denote the union of elements of $A$.

Let $n \geq 1, k \leq 2 n$ be fixed integers and $N=3 n-k$.

Let $E=\left\{e_{1}, \cdots, e_{N}\right\}$ be the set of labelled "edges" $e_{i}=\{2 i-1,2 i\}$. The set $E_{\frac{1}{2}}=\cup E=\{1, \cdots, 2 N\}$ is called the set of half-edges.

Definition 4.2 Let $D_{n, k}(M)$ denote the set of 4 -tuples $\Gamma=\left(U, T, E^{v}, \sigma\right)$, called labelled diagrams, which respect the following conditions:

- $E^{v} \subseteq E$ is the set of "visible edges", it labels the edges of the diagram. We let $E^{a}=E-E^{v}$ denote the set of "absent edges".

- The set $V=U \cup T$ of all vertices of $\Gamma$ is a partition of $\cup E^{v} \subseteq E_{\frac{1}{2}}$. 
- $U$ is the set of univalent vertices: it is a set of singletons, and $\sigma$ is an isotopy class of injections of $U$ into the interior of $M$.

- $T$ is the set of trivalent vertices, which are subsets of $E_{\frac{1}{2}}$ with three elements belonging to three different edges.

- $\quad \# V=2 n$ (which is equivalent to $\left.u_{\Gamma}=\# U=\# E^{a}+k\right)$.

- $\quad \Gamma$ is subprincipal (see Definition 1.7).

The identity is the only isomorphism of diagrams which preserves the labelling, so we will take the same notion of an isomorphism as before, which refers to the underlying unlabelled diagrams:

Definition 4.3 An isomorphism (or change of labelling) between two labelled diagrams $\Gamma=\left(U_{\Gamma}, T_{\Gamma}, E_{\Gamma}^{v}, \sigma_{\Gamma}\right)$ and $\Gamma^{\prime}=\left(U_{\Gamma^{\prime}}, T_{\Gamma^{\prime}}, E_{\Gamma^{\prime}}^{v}, \sigma_{\Gamma^{\prime}}\right)$ is a bijection from $\cup E_{\Gamma}^{v}$ to $\cup E_{\Gamma^{\prime}}^{v}$ which carries $E_{\Gamma}^{v}$ to $E_{\Gamma^{\prime}}^{v}, T_{\Gamma}$ to $T_{\Gamma^{\prime}}, U_{\Gamma}$ to $U_{\Gamma^{\prime}}$ and $\sigma_{\Gamma}$ to $\sigma_{\Gamma^{\prime}}$.

A configuration for a labelled diagram induces a map from $\cup E^{v}$ to $\mathbb{R}^{3}$, so the canonical map $\Psi$ can be written:

$$
\begin{aligned}
\Psi: C_{\Gamma} & \longrightarrow\left(S^{2}\right)^{E^{v}} \\
f & \longmapsto\left(\frac{f(2 i)-f(2 i-1)}{|f(2 i)-f(2 i-1)|}\right)_{e_{i} \in E^{v}}
\end{aligned}
$$

Then, we shall denote by $\Psi^{\prime}$ (but also sometimes by $\Psi$ by abuse of notation; fortunately, all ambiguities will be ineffective to the results) the map from $C_{\Gamma}^{\prime}=\overline{C_{\Gamma}} \times\left(S^{2}\right)^{E^{a}}$ to $\left(S^{2}\right)^{E}$ defined by the product of the previous $\Psi$ with the identity on $\left(S^{2}\right)^{E^{a}}$.

\subsection{Orientation of the configuration spaces}

We shall now define an orientation of the space $C_{\Gamma}^{\prime}$ depending on the orientation $o$ of $\Gamma$. It will be done by labelling all components of a natural local coordinate system of $C_{\Gamma}^{\prime}$ by the elements of $E_{\frac{1}{2}}$. These components are:

- One coordinate in $M$ for each univalent vertex, respecting the local orientation of $M$ given by the orientation of this vertex.

- The three standard components in $\mathbb{R}^{3}$ for each trivalent vertex.

- And for each absent edge we have the two components of a local coordinate system in $S^{2}$ with the standard orientation (i.e. where a basis $\left(x_{1}, x_{2}\right)$ at a 
point of $S^{2}$ is positively oriented when the vector product $x_{1} \wedge x_{2} \in \mathbb{R}^{3}$ points outwards $S^{2}$ ).

The coordinate in $M$ for a univalent vertex $\{x\}$ is labelled by $x$. The two components for each $e_{i} \in E^{a}$ are respectively labelled by $2 i-1$ and $2 i$.

As for the trivalent vertices, let us represent $o$ by a family of bijections $\left(b_{t}\right)_{t \in T}$ from each trivalent vertex $t$ to the set $\{1,2,3\}$. Then, for all $x \in t$, give the label $x$ to the $b_{t}(x)$-th component of $f(t)$ in $\mathbb{R}^{3}$.

Thus we have an orientation of $C_{\Gamma}^{\prime}$, which only depends on the orientation $o$ and the labelling of $\Gamma$. It is reversed when we change the orientation of one vertex or one edge; and the isomorphism induced by a permutation of $E$, which preserves the orientations of edges and vertices, also preserves the orientation of $C_{\Gamma}^{\prime}$. So, this construction is equivalent to first orienting $C_{\Gamma}$ by the same method with edges $e_{i}\left(i=1, \cdots, \# E^{v}\right)$, then taking the direct product with $\left(S^{2}\right)^{E^{a}}$.

For a labelled oriented diagram $(\Gamma, o)$, we can now define the integral

$$
I_{L}(\Gamma, o)=\int_{C_{\Gamma}^{\prime}} \Psi^{\prime *} \Omega=\int_{C_{\Gamma}} \Psi^{*} \Omega_{E^{v}}
$$

where $\Omega$ is the standard volume form on $\left(S^{2}\right)^{E}$ with total mass 1 , and $\Omega_{E^{v}}$ is the one on $\left(S^{2}\right)^{E^{v}}$.

Note that we have $I_{L}(\Gamma,-o)=-I_{L}(\Gamma, o)$ and that $I_{L}(\Gamma, o)$ is independent of the labelling of $\Gamma$ : exchanging two edges preserves both orientations of $C_{\Gamma}^{\prime}$ and $\left(S^{2}\right)^{E}$, and reversing one edge changes both orientations (precisely, it changes both the orientation of $C_{\Gamma}^{\prime}$ and the sign of the form $\Psi^{\prime *} \Omega$ ), because the antipodal map reverses the orientation of $S^{2}$.

So, the product $I_{L}(\Gamma)[\Gamma]$ is well-defined independently of $o$.

In all the following, we fix an arbitrary choice of orientations on the diagrams in $D_{n, k}(M)$, with no consequence on the final results.

\subsection{Interpretation of $Z$ in degree theory}

Let $\beta$ be a map from $D_{n, k}(M)$ to $\mathcal{A}_{n}^{k}(M)$ such that:

$$
\forall \Gamma \text { principal, } \sum_{\Gamma^{\prime} \in D_{n, k}(M), \Gamma^{\prime} \sim \Gamma} \beta\left(\Gamma^{\prime}\right)=\frac{[\Gamma]}{|\Gamma|}
$$


The standard example will be

$$
\beta(\Gamma)=\text { projection of } \frac{\left(N-e_{\Gamma}\right) !}{N ! 2^{e_{\Gamma}}}[\Gamma] \text { in } \mathcal{A}_{n}^{k}(M)
$$

but we need not restrict to it now.

Let $C^{\prime}$ be the following formal linear combination of spaces

$$
C^{\prime}=\sum_{\Gamma \in D_{n, k}(M)} \beta(\Gamma) C_{\Gamma}^{\prime}
$$

Here, $-C_{\Gamma}^{\prime}$ stands for $C_{\Gamma}^{\prime}$ with the opposite orientation. Let $\xi$ be the almost everywhere defined map with rational values in $\mathcal{A}_{n}^{k}(M)$ :

$$
\begin{aligned}
\xi:\left(S^{2}\right)^{E} & \longrightarrow \mathcal{A}_{n}^{k}(M) \\
x & \longmapsto \text { count of } \Psi^{-1}(x) \text { in } C^{\prime}
\end{aligned}
$$

According to the degree theory, this map can be extended into a locally constant map defined outside the union of the $\Psi\left(\partial C_{\Gamma}^{\prime}\right)$ for $\Gamma \in D_{n, k}(M)$. When a map is defined on a manifold without boundary, the number of preimages counted with signs is constant and called the degree of the map. In this article, we shall use a kind of generalised notion of "manifold without boundary", with a notion of gluing of faces that we shall define in the next section, such that only the faces which are not glued are relevant in the notion of a boundary. Now, we have

$$
Z_{n}^{k}=\int_{\left(S^{2}\right)^{E}} \xi \Omega
$$

So, $Z_{n}^{k}$ is generally not rational because the map $\xi$ is not constant on $\left(S^{2}\right)^{E}$. The aim of the following sections will be to prove Propositions 1.2 and 1.11 in the following way: we shall introduce other configuration spaces to complete $C^{\prime}$ so that the corresponding $\xi$ becomes constant.

Then, each of the expressions $Z^{0}$ of Proposition 1.2 and $Z_{n}^{k}$ of Proposition 1.11 will be equal to the degree of the map $\Psi$ defined on such a linear combination of configuration spaces which extends $C^{\prime}$.

Remark 4.7 We could generalise these constructions to other maps $\beta$ from $D_{n, k}(M)$ to any $\mathbb{Z}$-module. This possibility will be discussed in the Appendix. We shall see that in the case of a torsion-free module it gives no more invariants. The only interest is that it can improve the rationality result by means of another map $\beta$ which satisfies Formula (4.4) and optimised gluing conditions, but not (4.5). However, the improvement expressed in Remark 1.12 is not based on this method. 


\subsection{Yang's point of view}

Yang [Ya] introduced another interpretation of $Z$ in terms of degree theory, which is sometimes useful to avoid some computations of coefficients. Let us keep here Formula (4.5) for $\beta$.

The idea is to quotient the space $\left(S^{2}\right)^{E}$ by the group $\mathcal{G}$ of permutations and reversals of the edges, to obtain the space $B_{E}=\left(\mathbb{R P}^{2}\right)^{E} / \Sigma_{E}$ where $\Sigma_{E}$ is the permutation group of $E$. The group $\mathcal{G}$ also naturally acts on $C^{\prime}$, so that $\mathcal{G}$ commutes with $\Psi$ in these actions. Thus, the map $\xi$ is invariant under the action of $\mathcal{G}$ (for the same reason as the fact that $I_{L}(\Gamma)[\Gamma]$ did not depend on orientations) and induces a map defined on $B_{E}$.

To understand the quotient space $C^{\prime} / \mathcal{G}$, first note that the group of automorphisms of a diagram $\Gamma$ freely acts on $C_{\Gamma}$. Let $C_{\Gamma} /|\Gamma|$ denote the quotient space of $C_{\Gamma}$ by this action. It is almost everywhere identical to the space of all "configured diagrams" isomorphic to $\Gamma$, that is, unlabelled diagrams where the set $V$ of vertices is a subset of the space $\mathbb{R}^{3}$ and the univalent vertices belong to the image of $L$. Then $C^{\prime} / \mathcal{G}$ is topologically the union over all isomorphism classes of degree $n$ diagrams $\Gamma$ with $u_{\Gamma} \geq k$, of the product $C_{\Gamma} /|\Gamma| \times B_{E^{a}}$.

Now, the expression $Z_{n}^{k}$ can be interpreted as an integral on these quotients, but not of a $2 N$-form because some $I_{L}(\Gamma)$ can contribute even when the quotient space $C_{\Gamma} /|\Gamma|$ is not orientable (the simplest example is $\Gamma=\theta$ ). So we must replace the form $\Psi^{*} \Omega$ by the density $\left(\Psi^{*} \Omega \cdot\left(\right.\right.$ local orientation of $\left.C_{\Gamma}\right)$ ).

Let us drop the condition $u_{\Gamma} \geq k$ (or equivalently, take $k=2$ ); replace $C^{\prime}$ by the space $C$ constructed the same way as $C^{\prime} / \mathcal{G}$ but without the absent edges (it is the union of the $C_{\Gamma} /|\Gamma|$ for all degree $n$ diagrams $\Gamma$ up to isomorphism). Thus it is made of parts with different dimensions.

So, we can say that

$$
Z_{n}=\int_{C}\left(\Psi^{*} \Omega \cdot(\text { orientation }) \cdot[\Gamma]\right)_{l o c a l} .
$$

The whole integral $Z$ itself is defined in the same way with diagrams of all degrees.

Note that the rational coefficient disappears in the above integral formula. The advantage of this presentation is that it generally trivialises the problem of the handling of the rational coefficients of Formula (4.5). The bad point is that it gives no means to handle the sign of the density involved in the space $C$. 


\section{$5 \quad$ Ordinary gluings}

\subsection{Definition of a gluing}

In this subsection, we explain in all generality (for any map $\beta$ and without restricting to the set of configuration spaces we already defined) the notion of a gluing of a set of faces:

Each face $F$ of a configuration space $C_{\Gamma}^{\prime}$ contributes to a variation of $\beta(\Gamma)$ of the map $\xi$ while going across $\Psi(F)$. Here, $\Psi(F)$ is counted with possible multiplicities. We are going to define gluings for certain sets of faces, that is, objects whose existence ensure that the contributions of these faces to the discontinuity of $\xi$ at their images cancel. Note that the degenerated faces do not contribute to the discontinuity of $\xi$, thus no gluing is necessary for them.

Definition 5.1 A $\Psi$-isomorphism between two manifolds $\mathcal{M}_{1}$ and $\mathcal{M}_{2}$ with maps $\Psi$ from each of them to $\left(S^{2}\right)^{N}$, is a diffeomorphism $\varphi$ from $\mathcal{M}_{1}$ to $\mathcal{M}_{2}$ such that $\Psi \circ \varphi=\Psi$ on $\mathcal{M}_{1}$. A $\Psi$-isomorphism is said to be positive if it preserves their orientations.

Definition 5.2 A self-gluing of a face is a negative $\Psi$-automorphism of this face.

The existence of a self-gluing of a face clearly implies that this face does not contribute to the discontinuity of $\xi$. But self-gluings are quite exceptional and we shall use them only to eliminate some pathological cases of the other gluings.

In this section, we define gluings in the following way (but for the anomalous faces in the next section, it will be necessary to cut some faces into pieces).

Definition 5.3 A gluing of a set of faces $F(\Gamma, A)$ with coefficients $\beta(\Gamma)$ is a partition of this set such that in each class, all the faces are $\Psi$-isomorphic and verify the gluing condition

$$
\sum_{(\Gamma, A) \text { in the class }} \pm \beta(\Gamma)=0
$$

where the signs in front of $\beta(\Gamma)$ and $\beta\left(\Gamma^{\prime}\right)$ for two given pairs $(\Gamma, A)$ and $\left(\Gamma^{\prime}, A^{\prime}\right)$ will be equal iff each $\Psi$-isomorphism between $F(\Gamma, A)$ and $F\left(\Gamma^{\prime}, A^{\prime}\right)$ preserves their orientations inherited from the orientations of the spaces $C_{\Gamma}^{\prime}$ and $C_{\Gamma^{\prime}}^{\prime}$.

Note that if there are both a positive and a negative $\Psi$-isomorphism between two faces, then each face in the class is glued to itself, so the condition may be deleted. Otherwise, the condition for the signs in the above formula is consistent. 


\subsection{Gluing results for ordinary faces}

The aim of this section is to prove the following proposition.

Proposition 5.4 For the map $\beta$ defined by Formula (4.5), there exists a gluing of the set of ordinary, non-degenerated faces of the space $C^{\prime}$ defined by (4.6).

Proof We shall prove the following three lemmas in Subsection 5.4. They apply to the gluing of a combination $C^{\prime}$ as above but that can come from any map $\beta$ defined on the set of diagrams $D_{n, k}(M)$ with chosen orientations, and seen as extended to the set of the same diagrams with all orientations, according to the AS relation.

Lemma 5.5 The gluings of the type (c1) faces are ensured by the IHX' relations defined as follows: they are all possible relations of the following form between six subprincipal labelled diagrams that are identical outside the drawn part:

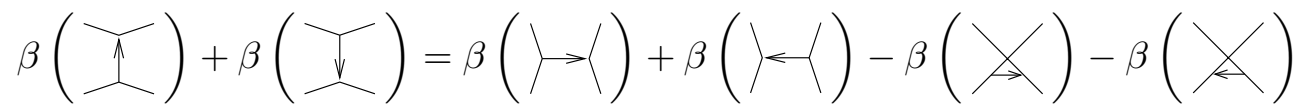

Lemma 5.6 The gluings of the type (c2) faces are ensured by the following $S T U$ ' relation between labelled diagrams that are identical outside the drawn part and such that all three unlabelled diagrams of the corresponding STU relation are subprincipal:

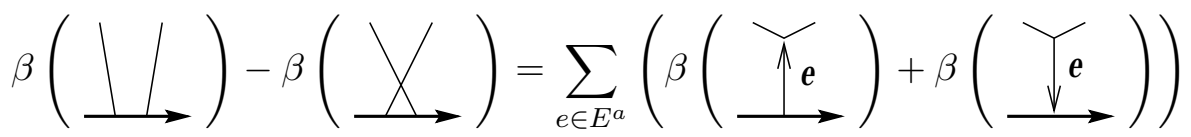

where $E^{a}$ is the set of absent edges of a diagram in the left-hand side of the relation (it can be empty).

If this $E^{a}$ is empty, this relation is verified in the case of Formula (4.5) because it is deduced from the STU relation and the fact that the missing diagram was forced to cancel in $\mathcal{A}_{n}^{k}(M)$.

Lemma 5.7 There is a gluing of the type (d) faces if $\beta$ is a constant on each isomorphism class of diagrams. 
The relations stated in the first two lemmas above will be fully used in the proofs to define the gluings, but the proof of the last lemma will not make full use of its assumption (that $\beta$ is constant on each isomorphism class) but only of a consequence of it, whose expression would be complicated to write and useless here. This possibility to weaken the condition can be used to improve the result on the denominators of the integral (see Appendix A.3).

Then, we finish the proof of Proposition 5.4 by the remark that the map $\beta$ defined by (4.5) satisfies the conditions of these lemmas.

\subsection{Gluing methods and orientations}

Now we are going to consider a general case of $\Psi$-isomorphism between two faces $F(\Gamma, A)$ and $F\left(\Gamma^{\prime}, A^{\prime}\right)$ of a same type ((c) or (d)). The problem will be to determine its sign $\varepsilon$.

We suppose that $\Gamma / A=\Gamma^{\prime} / A^{\prime}$ (as labelled diagrams), and that the $\Psi$-isomorphism preserves the projection onto $C_{\Gamma / A}$. So, the only transformations concern the graphs $A$ and $A^{\prime}$ and the spaces $C(A)$ and $C\left(A^{\prime}\right)$ (Notation 2.2), and if $A \cap U$ and $A^{\prime} \cap U^{\prime}$ are nonempty, they are mapped to the same line $l_{s}$ in the two identified configurations.

Moreover, let us suppose that $E_{A}=E_{A^{\prime}}$ (it will be the case for the gluings we shall consider except one of the two kinds of gluings for the STU' relation). This implies that $A$ and $A^{\prime}$ have the same respective numbers of univalent and trivalent vertices.

For convenience, we are going to label all vertices in $A$ independently of the labelling of the edges, starting with the univalent vertices (this means the elements of $U$ ), if they exist. We denote them by $x_{i}$ for $i \in X, X=\{0, \cdots, \# A-1\}$. Thus, $x_{0} \in U$ if $A \cap U \neq \emptyset$. By convention, we put the vertex $x_{0}$ at the origin of the space $R_{A}$. So $C^{A}$, which contains $C(A)$, is identified with the space of half lines in the vector space $\left(\mathbb{R}^{3}\right)^{X-\{0\}}$. We do the same for $\Gamma^{\prime}$ (so the univalent vertices in $A$ and $A^{\prime}$ are labelled by the same elements of $X$ ).

The neighbourhoods of the faces are locally identified (on open dense subsets, in natural ways with a definite limit near the interior of the face) with the fiber bundle $\mathcal{E}$ with base $C_{\Gamma / A}$ and fiber $\mathcal{E}_{s}$, where $\mathcal{E}_{s}$ is the space defined by: $\mathcal{E}_{s}=\left(\mathbb{R}^{3}\right)^{X-\{0\}}$ if $A \cap U=\emptyset$; and if $A \cap U \neq \emptyset, \mathcal{E}_{s}$ is the subspace of $\left(\mathbb{R}^{3}\right)^{X-\{0\}}$ consisting of the configurations which map $A \cap U$ to $l_{s}$, where $s$ is defined by the base point in $C_{\Gamma / A}$. 
We shall suppose that the bijection between the faces is defined over each element of $C_{\Gamma / A}$ by a linear transformation $\mathcal{L}$ of $\mathcal{E}_{s}$. If $A \cap U \neq \emptyset$, consider the set of vertices $A \cap U$ as a subset of $X$ : we shall suppose that $\mathcal{L}$ is the identity on the corresponding copies of $l_{s}$, and that the orientations of these vertices are preserved from $\Gamma$ to $\Gamma^{\prime}$.

Then, the bijection between the faces smoothly extends to a bijection of their neighbourhoods. As the orientation of each face of a space $\overline{C_{\Gamma}}$ was defined as coming from the orientation of $\overline{C_{\Gamma}}$, the sign $\varepsilon$ of the $\Psi$-isomorphism between $F(\Gamma, A)$ and $F\left(\Gamma^{\prime}, A^{\prime}\right)$ is given by the action on the neighbourhoods of the faces.

These neighbourhoods are both identified to the same space $\mathcal{E}$, but the orientation of this space $\mathcal{E}$ depends on the diagram. Therefore, we have

$$
\varepsilon=\operatorname{Sgn}(\operatorname{det} \mathcal{L}) \cdot \varepsilon^{\prime}
$$

where $\varepsilon^{\prime}$ is the sign of the change of orientation of $\mathcal{E}$ as orientations of the respective configurations spaces to which $\mathcal{E}$ was identified.

This $\varepsilon^{\prime}$ can be computed by comparing these orientations of $\mathcal{E}$ coming from the respective diagrams as defined in Subsection 4.2. The local coordinates, first labelled by the vertices, are then labelled by the half-edges. The labelled graphs $A$ and $A^{\prime}$ respectively define two bijections $b$ and $b^{\prime}$ between

$$
\left\{i \in X \mid x_{i} \in U\right\} \cup\left\{(i, 1),(i, 2),(i, 3) \mid i \in X, x_{i} \in T\right\}
$$

and the set $\cup A \subseteq E_{\frac{1}{2}}$ of half-edges which belong to a vertex in $A$.

Then, $\varepsilon^{\prime}$ is the signature of $b^{-1} b^{\prime}$.

\subsection{Proofs for the gluings}

\section{Proof of Lemma 5.5}

Each term of an IHX' relation corresponds to a type $(\mathrm{c} 1)$ face $F(\Gamma, e)$ where $\Gamma$ is the labelled diagram of the term, and $e$ is the edge involved in the relation.

If some type (c1) face does not fit into such a relation, it is because one other diagram of the IHX relation is not subprincipal, so its faces are degenerated. Since all faces of this unwritten IHX' relation are $\Psi$-isomorphic, they are all degenerated. This is why no relation is necessary for them.

So we have a partition of at least all non-degenerated type (c1) faces, and what we have to check is that this partition is a gluing, where the gluing condition is the IHX' relation. 
In the above constructions, we can always take $\mathcal{L}=\mathrm{Id}$, preserve the graph structure of $A$ but just use permutations of the set $E_{A}^{\prime}$. This provides $\Psi$ isomorphisms of faces with sign the signature of this permutation (it must be considered modulo the transpositions of two edges attached to the same vertex of $A$, because these transpositions induce the identity).

The verification of the signs in the IHX' relation is now easy.

\section{Proof of Lemma 5.7}

We consider now the non-degenerated type (d) faces.

According to Proposition 2.9, each edge in $E_{A}^{\prime}$ meets two edges (denoted as pairs of vertices) $e_{1}=\{x, y\}$ and $e_{2}=\{x, z\}$ in $E_{A}$. Suppose $x$ has a non-zero label. Let $\mathcal{L}$ be the map which maps (the position of) $x$ to $y+z-x$, and preserves the other vertices. This transformation has determinant -1 .

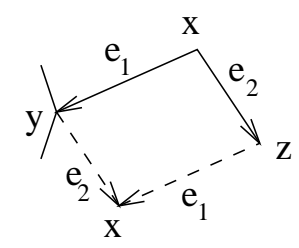

Then apply the only change of structure of $A$ which fits in with this transformation: it is a mere relabelling, which exchanges $e_{1}$ and $e_{2}$ and reverses them: if $e_{1}$ goes from $x$ to $y$ in $A$, then it goes from $z$ to $x$ in $A^{\prime}$. Suppose that these diagrams have the same orientation through this relabelling. This defines an even permutation of $\cup A$, so this $\Psi$-isomorphism is negative.

Now, here is a way to give a partition of these faces into pairs, which is a gluing as soon as $\beta$ is constant on each isomorphism class of diagrams with the same orientation.

Consider an (equivalence) class of pairs $(\Gamma, A)$ (concerned here) which are isomorphic by isomorphisms whose restriction to $\Gamma / A$ is the identity (thus $\Gamma / A$ is fixed as a labelled diagram). Then fix an arbitrary edge $e \in E_{A}^{\prime}$. By this choice, the diagrams in this class are grouped in pairs according to the above construction.

\section{Proof of Lemma 5.6}

First let us check that for each pair $(\Gamma, A)$ where $A$ is an edge or a pair of consecutive univalent vertices of $\Gamma$, the face $F(\Gamma, A)$ can be identified with the space $C_{\Gamma / A}^{\prime}$ : indeed, let us distinguish two cases. 
If $E_{A}=\emptyset$, it comes from the fact that the configuration of $A$ on this face in the compactified configuration space, is determined by the one of $\Gamma / A$ and the tangent map of $L$.

If $E_{A} \neq \emptyset$, we have $A \nsubseteq U$. This allows us to put the unique element of $E_{A}$ among the absent edges.

Therefore, all faces of a STU' relation (faces of the form $F(\Gamma, A)$ where $\# A=2$ and $\Gamma / A$ is identical to a fixed diagram with one bivalent vertex $\{A\}$ on $M$ ) are $\Psi$-isomorphic, thus, they are all degenerated when one is. This is true for $k=2$ when all faces of the STU relation are represented, thus, as the codimension does not depend on $k$ (which determines the number of absent edges), all faces of an STU' relation are degenerated when one face of the STU relation with unlabelled diagrams is.

This proves that aevery non-degenerated type (c2) face appears in an STU' relation.

Let us now study the signs of these $\Psi$-isomorphisms and check that the STU' relations are the gluing conditions. The sign of the $\Psi$-isomorphism between both left-hand side diagrams comes from the same method as in the IHX' gluing (here the space $\mathcal{E}_{s}$ has dimension 1 , $\operatorname{det} \mathcal{L}=1$ and $\varepsilon^{\prime}=-1$ because of the transposition).

Otherwise, let us study the sign of one $\Psi$-isomorphism between two faces corresponding to diagrams with a different number of univalent vertices. This is the exceptional case of sign study, but we shall treat it in a similar way to the other cases.

Consider the following diagrams, where each half-edge is marked with the name of a component in a local coordinate system of the neighbourhood of each face. Here $(x, y)$ stand for the spherical coordinates, and $u$ stands for the radial coordinate (outward normal vector to the sphere, thus inward normal vector to the face of the configuration space):
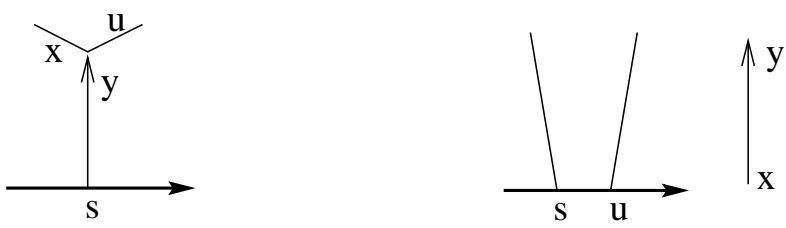

We see that these two labellings only differ by the transposition $(x, s)$, thanks to the way the standard orientation of $S^{2}$ was defined (Subsection 4.2). This proves that these two diagrams appear in the relation with different signs (or on different sides of the equality). 


\section{Anomaly}

\subsection{The anomaly as a degree of a map}

Recall that the non-degenerated types (a) and (a') faces are defined by a connected component $A$ of $\Gamma$, whose configurations map all univalent vertices of $A$ to a line $l_{s}$ directed by a tangent vector $s$ to $L$. To study them, we need to introduce a few definitions, where we replace $M$ by the non-compact line $\mathbb{R}$. This does not change the fundamental notions of diagrams and configuration spaces $\left(D_{n, k}(\mathbb{R})\right.$ is identical to $D_{n, k}(J)$ for instance).

Notation 6.1 Let $D_{n, k}^{W}$ be the set of connected elements of $D_{n, k}(\mathbb{R})$.

For all $s \in S^{2}$, let $l_{s}$ be the standard map $u \mapsto u s$ from $\mathbb{R}$ to the oriented axis of $\mathbb{R}^{3}$ with direction $s$. By an abuse of notation, $l_{s}$ will also mean this oriented axis.

For any $\gamma \in D_{n, k}^{W}$, let $W_{s}(\gamma)$ denote the quotient of $C_{\gamma}\left(l_{s}\right)$ by the translations and dilations of $\mathbb{R}^{3}$ which preserve the axis $l_{s}$.

Then, let $W(\gamma)$ denote the union of the $W_{s}(\gamma)$ for $s$ running over $S^{2}$.

We are going to give these spaces an orientation. First, let us orient the twodimensional Lie algebra of the group of translations and dilations which preserve $l_{s}$, by giving it a basis: take a first vector oriented to the positive translations of the oriented axis $l_{s}$, and a second vector oriented to the dilations of ratio greater than one.

Now, since the space $C_{\gamma}\left(l_{s}\right)$ is oriented by the method of Subsection 4.2, this orients the quotient space $W_{s}(\gamma)$, and therefore $W(\gamma)$ using the standard orientation of the base space $S^{2}$. There is no ambiguity, since all considered spaces are even-dimensional.

Then we define a map $\Psi_{\gamma}$ from $W(\gamma)$ to $\left(S^{2}\right)^{E^{v}}$, and a map $\Psi_{\gamma}^{\prime}$ from $W^{\prime}(\gamma)=$ $W(\gamma) \times\left(S^{2}\right)^{E^{a}}$ to $\left(S^{2}\right)^{E}$, exactly as before.

Now we are going to see that these spaces can be glued together according to the same formulas, except that there are no anomalous faces anymore.

Proposition 6.2 For the map $\beta$ defined by the same formula (4.5) on $D_{n, k}^{W}$, the linear combination of spaces

$$
W_{n, k}=\sum_{\gamma \in D_{n, k}^{W}} \beta(\gamma) W^{\prime}(\gamma)
$$

is completely glued, hence the union $\Psi$ of the $\Psi_{\gamma}^{\prime}$ admits a degree on it. 


\section{Proof of Proposition 6.2}

The method is to use the same proofs as those of Sections 4 and 5 in this new situation, where there are no equivalents of types (a), (a') and (e) faces (for the scale is relative and the diagrams are connected): we just have to check that the map $\Psi$ defined on the spaces $W(\gamma)$ factorises in the same way on the faces. It is very easy to do for the faces $F(\gamma, A)$ such that $A \subseteq T$ :

$$
F(\gamma, A)=W(\gamma / A) \times C(A) .
$$

The other faces can also be seen as fibered spaces with bases $W(\gamma / A)$ and fiber $W_{s}(A)$ : over each $y \in W(\gamma / A)$ we have the fiber $W_{s}(A)$ where $y \in W_{s}(\gamma / A)$.

The only new thing to prove is that if an STU' relation involves a non-connected diagram, then the faces are degenerated. This can easily be done by projecting the fiber over $S^{2}$ to the product of the corresponding fibers for the connected components: this product has one dimension less, corresponding to the relative dilations between the configurations of the two subdiagrams.

Definition 6.3 Let $\mathcal{D}^{W}$ be the set of connected elements of $\mathcal{D}(\mathbb{R})$. In other words, $\mathcal{D}^{W}$ is the set of isomorphism classes of diagrams in the $D_{n, 2}^{W}$ where $n$ runs over $\mathbb{N}$, together with a choice of arbitrary orientations. For all $\gamma \in \mathcal{D}^{W}$ we define the integral

$$
f_{\gamma}=\int_{W(\gamma)} \Psi_{\gamma}^{*} \Omega
$$

Then we define the anomaly to be

$$
\alpha=\sum_{\gamma \in \mathcal{D}^{W}} \frac{f_{\gamma}}{2|\gamma|}[\gamma] \in \mathcal{A}(\mathbb{R}) .
$$

Note that the degree $n$ part $\alpha_{n}$ is half the degree of the map $\Psi$ of the above proposition, when $\mathcal{A}_{n}^{k}(M)=\mathcal{A}_{n}(M)$. Moreover, $\alpha_{1}=\frac{[\theta]}{2}$. We are going to see that it is also the degree of a map defined on the quotient of $W_{n, k}$ by a symmetry, with still the same coefficients.

\subsection{Symmetry properties of the anomaly}

For any labelled oriented diagram $\gamma$ with support $\mathbb{R}$, let $\bar{\gamma}$ be the diagram obtained by reversing the injection $i: U \longrightarrow \mathbb{R}$ (by the composition with the 
involution $u \mapsto-u$ of $\mathbb{R}$ ), and transporting the orientations of univalent vertices with $U$ by this involution:

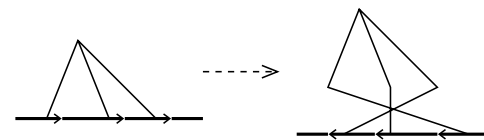

So the order of the vertices is changed. This defines a linear involution of each $\mathcal{A}_{n}(\mathbb{R})$ and an automorphism of $\mathcal{A}(\mathbb{R})$.

We say that a element of $\mathcal{A}(\mathbb{R})$ is $S^{1}$-even if it is preserved by this involution. It is unknown whether all elements of $\mathcal{A}(\mathbb{R})$ are $S^{1}$-even. Now, let $-\gamma$ denote the labelled diagram obtained from $\gamma$ by reversing all its edges and preserving the vertex orientations. We obviously have $[-\gamma]=[\gamma]$ because these diagrams are isomorphic.

We have the two properties:

Lemma 6.4 (i) For all $s \in S^{2}, \gamma \in D_{n, k}^{W}$, there is a negative $\Psi$-isomorphism between $W_{s}(\gamma)$ and $W_{-s}(\bar{\gamma})$.

(ii) the anomaly is $S^{1}$-even $(\bar{\alpha}=\alpha)$.

Lemma 6.5 (i) For all $s \in S^{2}$ and $\gamma \in D_{n, k}^{W}$, there is a $\Psi$-isomorphism between $W_{s}(\gamma)$ and $W_{-s}(-\gamma)$ with sign $(-1)^{n}$.

(ii) The anomaly cancels at even degrees.

\section{Proof of Lemma 6.4}

The $\Psi$-isomorphism that we take from $W_{s}(\gamma)$ to $W_{-s}(\bar{\gamma})$ is the identity on configurations as maps from $V$ to $\mathbb{R}^{3}$. The sign comes from the fact that we quotient the configurations by the action of the oriented group of dilations and translations along the axis $l_{s}$ which is reversed. Now, this map preserves the orientation between $W(\gamma)$ and $W(\bar{\gamma})$ because the antipodal map on the base space $S^{2}$ also reverses its orientation. Thus, $f_{\gamma}=f_{\bar{\gamma}}$.

\section{Proof of Lemma 6.5}

The $\Psi$-isomorphism we take between $W_{s}(\gamma)$ and $W_{-s}(-\gamma)$ is the central symmetry on configurations. Note that this symmetry transports the orientation of $l_{s}$ and the orientations of univalent vertices. Thus, the orientation changes come from the geometrical symmetry for each trivalent vertex, and the reversal of each edge in the diagram. So, the sign is $(-1)^{n}$ because of the formula $\# E-\# T=\operatorname{deg} \gamma=n$. Finally, the antipodal map on the base space and the isomorphism $-\gamma \sim \gamma$ give $f_{\gamma}=(-1)^{n+1} f_{-\gamma}=(-1)^{n+1} f_{\gamma}$ : $f_{\gamma}$ cancels for even $n$. 
Notation 6.6 For $n$ odd, the $\Psi$-isomorphism between $W_{s}(\gamma)$ and $W_{-s}(-\gamma)$ defines an orientation-preserving involution of $W_{n, k}$. We let $\widetilde{W}_{n, k}$ be the quotient space of $W_{n, k}$ by this involution, and let $\widetilde{D}_{n, k}^{W}$ be the quotient of $D_{n, k}^{W}$ by the involution $\gamma \mapsto-\gamma$. It lets no diagram fixed because it changes the half-edge of the first univalent vertex on $\mathbb{R}$. Thus,

$$
\widetilde{W}_{n, k}=\sum_{\gamma \in \widetilde{D}_{n, k}^{W}} \beta(\gamma) W^{\prime}(\gamma)
$$

We can note that the image of $\alpha_{n}$ in $\mathcal{A}_{n}^{k}(\mathbb{R})$ is equal to the degree of the map $\Psi$ defined on $\widetilde{W}_{n, k}$.

\subsection{The labelled generalised diagrams and their configuration spaces}

Now we are going to define the additional configuration spaces to complete $C^{\prime}$ in order to glue all the faces and define the degree of $\Psi$ in the case of a link.

First, identify $M$ with $X \times S^{1}$ where $X$ is a finite set. Then choose a family of smooth maps $\varphi_{m}: D^{2} \longrightarrow S^{2}$ for $m \in X$, where $D^{2}$ is the disc and each $\varphi_{m}$ coincides with the tangent map to the link $L$ on the boundary $\{m\} \times S^{1}$ of $D^{2}$. Here we equip $D^{2}$ with the opposite orientation to the usual one. So, the basis (tangent oriented by $S^{1}$, outward normal) is direct.

Notation 6.7 For all $\gamma \in D_{n, k}^{W}$ and $m \in X$, let

$$
\mathcal{W}_{m}(\gamma)=\varphi_{m}^{*} W(\gamma)
$$

be the fibered space over $D^{2}$ which is the union of the $W_{\varphi_{m}(x)}(\gamma)$ for $x \in D^{2}$, and equip it with the product orientation.

In the same way as in Subsection 4.1, we let $E=\left\{e_{1}, \cdots, e_{N}\right\}$ be the set of labelled "edges" $e_{i}=\{2 i-1,2 i\}$. We recall the formula $k=3 n-N$.

Now fix $N$ and let $n$ and $k$ vary for the sets of diagrams $D_{n, k}^{W}$ and $\widetilde{D}_{n, k}^{W}$.

Let $D_{N}^{W}$ denote the union of the $D_{n, 3 n-N}^{W}$ for all $n$. And let $\widetilde{D}_{N}^{W}$ denote the union of the $\widetilde{D}_{n, 3 n-N}^{W}$ for all $n$ such that $n$ is odd.

In fact, these unions concern the $n$ such that $2 n-1 \leq N$, for connected diagrams of higher degrees would have more edges. 
Definition 6.8 A labelled generalised diagram with support $M$ is the data of a decomposition of $E$ into a disjoint union

$$
E^{v} \cup E^{a} \cup E^{W}
$$

together with:

- a labelled diagram $\Gamma_{0}$ with support $M$ such that its set of visible edges is $E^{v}$, in the sense of Definition 4.2;

- a set $Y$ of diagrams in $D_{N}^{W} \sqcup \widetilde{D}_{N}^{W}$ such that their sets of visible edges form a partition of $E^{W}$, together with a map $\gamma \mapsto m(\gamma)$ from $Y$ to $X$. We let $Y_{1}=Y \cap D_{N}^{W}$ and $Y_{2}=Y \cap \widetilde{D}_{N}^{W}$.

The degree of a labelled generalised diagram is the sum of the degrees of $\Gamma_{0}$ and all the diagrams $\gamma \in Y$. Let $D_{n, 3 n-N}^{g}$ denote the set of these generalised diagrams of degree $n$. An arbitrary orientation is chosen on each of them.

Note that $D_{n, k}(M) \subseteq D_{n, k}^{g}$. Indeed, $D_{n, k}(M)$ is the set of generalised diagrams $\Gamma \in D_{n, k}^{g}$ such that $Y=\emptyset$, or equivalently $E^{W}=\emptyset$.

Definition 6.9 Let the configuration space of a generalised diagram be

$$
C_{\Gamma}=C_{\Gamma_{0}} \times \prod_{\gamma \in Y_{1}} \mathcal{W}_{m(\gamma)}(\gamma) \times \prod_{\gamma \in Y_{2}} W(\gamma)
$$

with the product orientation. This is again completed with the help of the absent edges:

$$
C_{\Gamma}^{\prime}=C_{\Gamma} \times\left(S^{2}\right)^{E^{a}}
$$

The product orientation is well-defined because all spaces are even-dimensional. It is reversed when we change the orientation of a single vertex. The canonical map $\Psi$ extends to the configuration spaces of generalised diagrams in the obvious way.

\subsection{Gluing the anomalous faces}

Proposition 6.10 Let $p$ be a fixed map from $X$ to $\mathbb{Z}$. We extend $\beta$ to generalised diagrams according to the same formula (4.5) where now $e_{\Gamma}=$ $\#\left(E^{v} \cup E^{W}\right)$ and the images $[\Gamma] \in \mathcal{A}(M)$ of the generalised diagrams $\Gamma$ are defined by

$$
[\Gamma]=\left[\Gamma_{0}\right] \prod_{\gamma \in Y_{1}}[\gamma]^{(m(\gamma))} \times \prod_{\gamma \in Y_{2}} p_{m(\gamma)}[\gamma]^{(m(\gamma))} .
$$


Then, the canonical map $\Psi$ defined on the linear combination

$$
\sum_{\Gamma \in D_{n, k}^{g}} \beta(\Gamma) C_{\Gamma}^{\prime}
$$

admits a degree which belongs to the integral lattice generated by the elements $\beta(\Gamma)$ in the space $\mathcal{A}_{n}^{k}(M)$ where $\Gamma$ runs over the principal diagrams in $\mathcal{D}_{n}(M)$.

\section{Proof Ordinary gluings}

The ordinary gluings of $\Gamma_{0}$ and all the $\gamma \in Y$ are an immediate consequence of Section 5 and Proposition 6.2. Here the STU' relation always involves the set $E^{a}$ of absent edges of the generalised diagram, given in Definition 6.8.

\section{Anomaly gluings}

Now we are going to glue together the following two types of faces.

- The types (a) and (a') faces for the subdiagram $\Gamma_{0}$ of a generalised diagram;

- The faces induced by the boundary of $D^{2}$ on some $\mathcal{W}_{m(\gamma)}(\gamma)$.

These faces are glued in pairs in an obvious way, where one face of the first type is glued to a piece of a face of the second type delimited by the positions of the univalent vertices of $\Gamma_{0}$ on the corresponding component $\{m\} \times S^{1}$ of $M$.

Now we have to check that the two orientations are opposite in any case. The orientation of $D^{2}$ has been defined by the basis (tangent oriented by $S^{1}$, outward normal), whereas for the type (a) or (a') faces, we must consider the orientation of the Lie algebra by which we quotiented, when we defined the orientation of the spaces $W_{s}(\gamma)$ : we took the basis (translations in the sense of $s$, growing dilations). But $s$ represents the generic tangent vector to $L$ with the positive orientation, whereas the growing dilations point inward to the configuration space.

\section{Generators}

By definition, the degree of $\Psi$ is equal to the count of preimages of a generic point with coefficients $\beta(\Gamma)$. So, it belongs to the integral lattice generated by the $\beta(\Gamma)$ when $\Gamma$ runs over the generalised diagrams such that $\Psi\left(C_{\Gamma}\right)$ has nonempty interior. These diagrams are principal, as this necessary condition remains true generalising Proposition 1.8 (but the converse is false: a principle diagram $\gamma$ can give an empty interior for a $W(\gamma)$ ). Then, we may keep the only non-generalised diagrams in this list, thanks to Formula (6.11). 


\subsection{Proofs of Propositions 1.2 and 1.11}

Notation 6.12 For each $m \in X$, define the integral

$$
I_{m}=\int_{D^{2}} \varphi_{m}^{*} \omega
$$

Lemma 6.13 The integrals on the spaces $\mathcal{W}_{m}(\gamma)$ obey the identity

$$
\int_{\mathcal{W}_{m}(\gamma)} \Psi_{\gamma}^{*} \Omega=I_{m} f_{\gamma}
$$

This comes from the invariance of the space $W(\Gamma)$ and of the volume form $\Omega$ under rotations of $S^{2}$.

Proposition 6.14 The expression deg $\Psi$ of Proposition 6.10 is the projection in $\mathcal{A}_{n}^{k}(M)$ of

$$
Z \prod_{m \in X} \exp \left(\left(p_{m}+2 I_{m}\right) \alpha^{(m)}\right) .
$$

The proof of this proposition is just a matter of calculation. Let $\mathcal{D}_{n, k}^{g}$ be the set of isomorphism classes of generalised diagrams in $D_{n, k}^{g}$. It is the set of classes of degree $n$ generalised diagrams $\Gamma$ such that $e_{\Gamma} \leq N$.

We have

$$
\begin{aligned}
\operatorname{deg} \Psi & =\sum_{\Gamma \in D_{n, k}^{g}} \frac{\left(N-e_{\Gamma}\right) !}{N ! 2^{e_{\Gamma}}} I_{L}(\Gamma)[\Gamma] \\
& =\sum_{\Gamma \in \mathcal{D}_{n, k}^{g}} \frac{I_{L}\left(\Gamma_{0}\right)\left[\Gamma_{0}\right]}{|\Gamma|} \prod_{\gamma \in Y_{1}} I_{m(\gamma)} f_{\gamma}[\gamma]^{(m(\gamma))} \times \prod_{\gamma \in Y_{2}} p_{m(\gamma)} f_{\gamma}[\gamma]^{(m(\gamma))} .
\end{aligned}
$$

For any generalised diagram $\Gamma, m \in X$ and $\gamma \in \mathcal{D}^{W}$, let $\varepsilon_{\gamma, m}^{\Gamma}$ denote the number of copies of $\gamma$ colored by $m$ in the set $Y_{1}$ of $\Gamma$, and let $\eta_{\gamma, m}^{\Gamma}$ denote its number in the set $Y_{2}$.

Then, the number of automorphisms of $\Gamma$ is:

$$
|\Gamma|=\left|\Gamma_{0}\right| \prod_{\substack{\gamma \in \mathcal{D}^{W} \\ m \in X}}|\gamma|^{\varepsilon_{\gamma, m}^{\Gamma}}\left(\varepsilon_{\gamma, m}^{\Gamma}\right) !(2|\gamma|)^{\eta_{\gamma, m}^{\Gamma}}\left(\eta_{\gamma, m}^{\Gamma}\right) !
$$

So we have

$$
\operatorname{deg} \Psi=\sum_{\Gamma \in \mathcal{D}_{n, k}^{g}} \frac{I_{L}\left(\Gamma_{0}\right)\left[\Gamma_{0}\right]}{\left|\Gamma_{0}\right|} \prod_{\substack{\gamma \in \mathcal{D}^{W} \\ m \in X}} \frac{\left(I_{m}\right)^{\varepsilon_{\gamma, m}^{\Gamma}}}{\varepsilon_{\gamma, m}^{\Gamma} !}\left(\frac{f_{\gamma}[\gamma]^{(m)}}{|\gamma|}\right)^{\varepsilon_{\gamma, m}^{\Gamma}+\eta_{\gamma, m}^{\Gamma}} \frac{1}{\eta_{\gamma, m}^{\Gamma} !}\left(\frac{p_{m}}{2}\right)^{\eta_{\gamma, m}^{\Gamma}} .
$$


This is the image in $\mathcal{A}_{n}^{k}(M)$ of the degree $n$ part of

$$
\left(\sum_{\Gamma_{0} \in \mathcal{D}} \frac{I_{L}\left(\Gamma_{0}\right)\left[\Gamma_{0}\right]}{\left|\Gamma_{0}\right|}\right) \prod_{m \in X} \exp \left(\sum_{\gamma \in \mathcal{D}^{W}} \frac{f_{\gamma}[\gamma]^{(m)}}{|\gamma|}\left(I_{m}+\frac{p_{m}}{2}\right)\right)
$$

which is nothing but

$$
Z \prod_{m \in X} \exp \left(\left(p_{m}+2 I_{m}\right) \alpha^{(m)}\right)
$$

Lemma 6.15 For all $m, I_{L}\left(\theta_{m}\right)+2 I_{m} \in \mathbb{Z}$.

We can restrict ourselves to one fixed component $m$ of $M$, thus to the case of a knot. Apply Proposition 6.14 to the case $n=N=1$ : the degree 1 part of $Z \exp \left(\left(p_{m}+2 I_{m}\right) \alpha\right)$ is $I_{L}(\theta) \frac{[\theta]}{2}+\left(p_{m}+2 I_{m}\right) \frac{[\theta]}{2}$. It is the degree of a map where the coefficient is $\beta(\theta)=\frac{[\theta]}{2}$. Thus, $I_{L}\left(\theta_{m}\right)+2 I_{m}$ is an integer.

\section{Proof of Proposition 1.2}

The above constructions work for any map $p$ from $X$ to $\mathbb{Z}$. Now, let $p_{m}=$ $-\left(I_{L}\left(\theta_{m}\right)+2 I_{m}\right)$. In an isotopy, we let the maps $\varphi_{m}$ vary continuously, so the integer $p_{m}$ is constant for any $m$.

Now let

$$
Z^{0}=Z \prod_{m \in X} \exp \left(\left(p_{m}+2 I_{m}\right) \alpha^{(m)}\right)
$$

Its part of each degree is the degree of a map, thus it is constant in each isotopy class. We have $p_{m}+2 I_{m}=-I_{L}\left(\theta_{m}\right)$. We conclude that

$$
Z=Z^{0} \prod_{m \in X} \exp \left(I_{L}\left(\theta_{m}\right) \alpha^{(m)}\right)
$$

\section{Proof of Proposition 1.11}

We know that $I_{L}\left(\theta_{m}\right)+2 I_{m}$ is an integer. If we suppose that $I_{L}\left(\theta_{m}\right)$ is an integer, then we can let $p_{m}=-2 I_{m}$. Thus, for any fixed $n$ and $k, Z_{n}^{k}$ is equal to $\operatorname{deg} \Psi$. The conclusion comes from Proposition 6.10 .

Remark 6.16 It is well-known that for a $\operatorname{knot}\left(I_{m}=I, \theta_{m}=\theta, \varphi_{m}=\varphi\right)$, the Gauss integral $I_{L}(\theta)$ is some sort of measure of the framing of the knot, that is, the linking number of the knot with a parallel curve. Now we have proved that $I_{L}(\theta)+2 I$ is equal to an integer. So one can wonder how to construct 
geometrically the parallel curve with framing $I_{L}(\theta)+2 I$ from the map $\varphi$. Here is the answer:

Consider the fibre bundle over $D^{2}$, with fiber over $x \in D^{2}$ the normal plane to the vector $\varphi(x)$. Since $D^{2}$ is homotopically trivial, there is a unique trivialisation of this bundle up to homotopy. This gives a homotopy class of trivialisation of the bundle over the boundary $S^{1}$ of $D^{2}$, which is the normal bundle to the knot. Thus, it gives our parallel curve up to isotopy.

\section{Vanishing of the anomaly in degree 3}

In this section we are going to prove that the degree 3 part of $\alpha$ vanishes, although the integrals $f_{\gamma}$ that occur in its expression do not individually cancel.

It is easy to see that the degree 3 diagrams in $\mathcal{D}^{W}$ are $\Lambda \Lambda, \Omega$, and $\boldsymbol{\varnothing}$. They have no automorphisms other than the identity. We also easily find, when

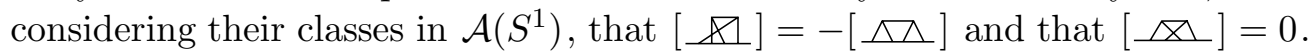
So, what we have to prove is that $f_{\triangle \Lambda}=f_{\nexists \Perp}$.

Put the following labelling on the diagrams $\Lambda \lambda$ and $\not \Perp$ :
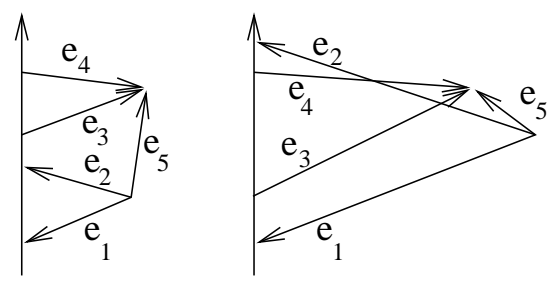

Now we are going to describe the regions $\Psi\left(C_{\Lambda_{\Lambda}}\right)$ and $\Psi\left(C_{\beth_{\perp}}\right)$ of $\left(S^{2}\right)^{E}$, $E=\left\{e_{1}, \cdots, e_{5}\right\}$. They are the sets of configurations of $E$ in $S^{2}$ which verify the two conditions:

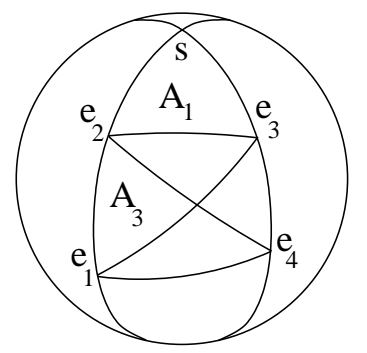

(1) The points $e_{1}, e_{2}, e_{3}, e_{4}$ form a "square", that is, they obey the four conditions obtained by circular permutations of this one: the points $e_{3}$ and $e_{4}$ are on the same side of the vector plane $\left(e_{1}, e_{2}\right)$ which contains $e_{1}$ and $e_{2}$. 
Indeed, considering the vector $s$ orienting $l_{s}$ as the north pole, we can find it on the picture up to sign as the intersection of $\left(e_{1}, e_{2}\right)$ and $\left(e_{3}, e_{4}\right)$. We must have $e_{1}$ and $e_{2}$ on the same meridian (half-circle from $s$ ), and the same for $e_{3}$ and $e_{4}$. This gives two of the above four conditions. The two other conditions are equivalent expressions of the fact that, while the information on the sign of $s$ is given by the orientation of any of these meridians, respectively from $e_{1}$ to $e_{2}$ and from $e_{4}$ to $e_{3}$, these two informations agree.

(2) The point $e_{5}$ is in the region $A_{1}$ in the case of $\triangle \wedge$, and in the region $A_{3}$ in the case of $\not \mathbb{L}$.

Indeed, before fixing $e_{5}$, the values on the sphere of the $e_{i}$ for $i \leq 4$ determine $l_{s}$ and the shape of each of the two halves of the diagram $\left(\left\{e_{1}, e_{2}\right\}\right.$ on the one hand, $\left\{e_{3}, e_{4}\right\}$ on the other hand) up to independant translations and dilations preserving $l_{s}$.

Then, in the case of $A_{1}$ (image of $\triangle \backslash$ ), the vector $e_{5}$ must be equal to $e_{2}+e_{3}+\lambda s$ for some $\lambda>0$ and where each $e_{i}$ is determined only up to a positive scalar multiplication. Therefore it means that $e_{5}$ is in the region $A_{1}$.

The case of $A_{3}$ (image of $\nexists_{1}$ ) is defined by the fact that $e_{2}$ is above $e_{4}$ and $e_{3}$ is above $e_{1}$. Therefore, $e_{5}$ is of the form $e_{2}+e_{4}-\lambda s$ on the one hand, $e_{1}+e_{3}+\lambda^{\prime} s$ on the other hand. This gives the region $A_{3}$.

Note that there are two possible orientations for the square $\left(e_{1}, e_{2}, e_{3}, e_{4}\right)$, corresponding to the two sides of the vector plane $\left(e_{1}, e_{2}\right)$ where the points $e_{3}$ and $e_{4}$ can be. This splits the configuration spaces into two subsets. We can restrict the study to one of them, since the other one is obtained from it by the central symmetry: we know that this symmetry preserves the integral according to Lemma 6.5. On the interior of each of these subsets, $\Psi$ is a diffeomorphism.

To show the equality of the integrals on these two areas, we shall first show the equality of signs, then the equality of absolute values.

To prove the equality of signs, let us see the areas $A_{1}$ and $A_{3}$ as two regions of the biangle $B\left(e_{1}, e_{2}, e_{3}, e_{4}\right)$ with one side containing $e_{1}$ and $e_{2}$, and the other side containing $e_{3}$ and $e_{4}$. Let us extend the map $\Psi$ to a larger "configuration space" which contains $C \triangle \wedge$ and $C \triangle \downarrow$ as open subsets, and in which one can go continuously from one to the other.

This larger configuration space is defined as follows. It is the quotient by dilations and translations of the set of maps from $V$ to $\mathbb{R}^{3}$ such that:

- It is injective on each edge. 
- It is not contained in a plane.

- All univalent vertices are on a straight line.

- On this line, the respective positions $z_{i}$ of the ends of $e_{i}(1 \leq i \leq 4)$ verify $\left(z_{2}-z_{1}\right)\left(z_{4}-z_{3}\right)>0$.

Its image by $\Psi$ is the region of $\left(S^{2}\right)^{E}$ defined by the conditions that $e_{1}, e_{2}$, $e_{3}, e_{4}$ form a square, and that $e_{5}$ is inside the biangle $B\left(e_{1}, e_{2}, e_{3}, e_{4}\right)$. It is an imbedding, thus its jacobian keeps its sign on each connected component.

So, as each connected component meets both $C_{\triangle \Lambda}$ and $C_{\Perp \Perp}$, we have the same signs on them.

Finally, to prove that the integrals on $C \wedge \lambda$ and $C \triangle \Perp$ have the same absolute value, we are going to show that the system of inequalities delimiting their image (positions of the $e_{i}$ such that $e_{5}$ is in $A_{1}$, resp. $A_{3}$ relatively to the others) are similar. Their similarity comes from this remark:

Denoting for three vectors $s_{1}, s_{2}, s_{3}$ of $\mathbb{R}^{3},\left[s_{1}, s_{2}, s_{3}\right]=\operatorname{sign}\left(\operatorname{det}\left(s_{1}, s_{2}, s_{3}\right)\right)$, the condition that $\left(e_{1}, e_{2}, e_{3}, e_{4}\right)$ is a square is expressed by

$$
\left[e_{1}, e_{2}, e_{3}\right]=\left[e_{1}, e_{2}, e_{4}\right]=\left[e_{1}, e_{3}, e_{4}\right]=\left[e_{2}, e_{3}, e_{4}\right] .
$$

Therefore it is equivalent to saying that $\left(e_{2}, e_{3},-e_{1},-e_{4}\right)$ is a square.

Now, the system of inequalities for $A_{3}$ is that $\left(e_{1}, e_{2}, e_{3}, e_{4}\right)$ is a square and $e_{5}$ is inside this square, in the triangle limited by the diagonals and one fixed side $\left(e_{1}, e_{2}\right)$.

The one for $A_{1}$ is that $\left(e_{2}, e_{3},-e_{1},-e_{4}\right)$ is a square and $e_{5}$ is inside this square, in the triangle limited by the diagonals and the side $\left(e_{2}, e_{3}\right)$.

\section{$8 \quad Z^{0}$ is a universal Vassiliev invariant}

We are going to see that the invariant $Z^{0}$ is a universal Vassiliev invariant. But there is a little problem: we have to choose between three possible conventions. Either we study $Z$ as a Vassiliev invariant of framed links with framings $I_{L}\left(\theta_{m}\right)$ (that can be supposed to be integers), or we look at $Z^{0}$ as an invariant of zeroframed links (but it is rather artificial as this framing must be corrected at each desingularisation), or we just consider the common image of all this under the quotient of $\mathcal{A}(M)$ by the ideal generated by $\theta$, as an invariant of unframed links. We shall consider here the question in terms of the first approach. 
Let us recall the defining property of universal Vassiliev invariants. A map $\mathcal{I}$ from the set of isotopy classes of framed links $L: M \hookrightarrow \mathbb{R}^{3}$ to $\mathcal{A}(M)$ is a universal Vassiliev invariant if: for any integer $n$ and any singular oriented link with $n$ double points, that is, an immersion $L$ of $M$ into $\mathbb{R}^{3}$ which is an imbedding except for a set $X$ of $n$ points which have two preimages, the alternate sum of desingularised links

$$
\sum_{\zeta: X \longrightarrow\{-1,1\}}\left(\prod_{i} \zeta(i)\right) \mathcal{I}\left(L_{\zeta}\right)
$$

is zero in all degrees $<n$, and is equal in degree $n$ to the chord diagram defined by the positions of singular points in $M$. Here, $L_{\zeta}$ is the link obtained from $L$ by replacing the singularities by the crossings with signs defined by $\zeta$.

Let us prove this property of $Z$ where the framings are given by the $I_{L}\left(\theta_{m}\right)$, considering that the movement of desingularisation is infinitesimal.

Consider $Z$ in terms of configured diagrams as described in Subsection 4.4. The spaces $C$ of the different desingularised links are in correspondence except at places where some part of graph connects the two strands together inside the neighbourhood of a desingularisation. Then, the alternate sum cancels all contributions of the parts of the $C$ in which there is not the presence of such a part of graph near every singular point.

The configured diagrams which do contain such a part of graph near every singular point must have degree $\geq n$ : indeed, they must have at least one univalent vertex on each strand of the singular points, so globally at least $2 n$ univalent vertices. The only degree $n$ diagram which has the above property is precisely the expected chord diagram.

Now, the alternate sum of integrals on these configured chord diagrams is easily computed: at every neighbourhood of a singular point, the direction of the chord runs through a hemisphere limited by the tangent plane at the singularity for each desingularised diagram. The difference gives the surface of the whole sphere which is equal to 1 according to our definitions.

\section{A Appendix: Changing the map $\beta$}

In the Appendix, we study the possibility of choosing a map $\beta$ different from Formula (4.5). It is of no usage in the rest of this article. 


\section{A.1 Weakening the gluing conditions}

Finding other maps instead of (4.5) that still satisfies a gluing to obtain a result like in Proposition 6.10 is something difficult. We shall not see general solutions but we shall mention some elements of the problem.

The first aspect of the problem is to make a complete list of configuration spaces whose image have codimension 0 or 1 . It seems unlikely that something more could be done than we did, except for the $W(\gamma)$. As concerns the question of finding possible other configuration spaces to complete the list, Dylan Thurston [Th] proposed to use spaces constructed in the same way as our $\mathcal{W}_{m}(\gamma)$ where $D^{2}$ and $\varphi$ are replaced by $C_{\theta}$ and the map $\Psi$ defined on it. But such spaces do not need to be introduced because they can always be replaced by a suitable combination of the $\mathcal{W}_{m}(\gamma)$ and $W(\gamma)$ as we did. I do not know if one can introduce usefully other spaces.

The second aspect of the problem is to determine which faces are degenerated. One can sometimes deduce that a face is degenerated from the fact that it is $\Psi$-isomorphic to a face of another configuration space with a codimension $\geq 2$ image.

We can generalise Lemma 3.5 to the fact that a type (d) face is degenerated if $\exists B \subseteq A \cap T$, \# $\left(E_{B}^{\prime} \cap E_{A}\right)=1$, or if $A$ is not connected.

It should be possible to find more degenerated type (a) faces, for example the case when at least 3 trivalent vertices are related to 2 univalent vertices each. (The corresponding $W(\gamma)$ has codimension 1 image).

The third aspect is to make a list of all possible $\Psi$-isomorphisms between nondegenerated faces, to enlarge the equivalence relation between faces, and thus to reduce the number of gluing conditions, so that more maps $\beta$ may satisfy them.

We now recall the gluing conditions and give possible ways of weakening them:

(1) The STU' relation (Lemma 5.3): no suggestion.

(2) The IHX' relation (Lemma 5.2), that we can replace by the following weaker IHX" relation: a IHX" relation is the sum of the IHX' relations obtained the following way: we fix the labelled diagram $\Gamma / e$ where $e \subseteq T$ is the edge involved, and we let $e$ be replaced by each absent edge.

(3) The real conditions to glue the type (d) faces are far less constraining than to impose $\beta$ to be constant on isomorphism classes of diagrams. With one bivalent vertex on $A$ the weaker condition is easy to write as there is only one 
way to group in pairs the faces of labelled diagrams for the $\Psi$-isomorphism we wrote. With more bivalent vertices it is more complicated as the same construction gives many possible $\Psi$-isomorphisms; moreover, all permutations of $E_{A}^{\prime}$ may be used. In the case of $A \subseteq T$ with $\# E_{A}^{\prime}=3$, the position of $A$ in the diagram $\Gamma / A$ may be changed. But these weaker conditions are not easy to make explicit.

(4) As for the anomaly, we could quotient the configuration spaces of generalised diagrams by all $\Psi$-isomorphisms used in Lemmas 6.4 and 6.5. Then, a sufficient condition for the map $\beta$ defined on ordinary diagrams to be extendable to generalised diagrams in a way which satisfies the resulting gluing conditions is that: for all diagrams obtained by inserting a connected $\gamma$ into a fixed diagram $\Gamma$ at different places of a fixed component of $M$, the expression

$$
\beta(\Gamma, \gamma)+(-1)^{\operatorname{deg} \gamma+1} \beta(\Gamma,-\bar{\gamma})
$$

does not depend on the place where $\gamma$ is inserted. It may not be necessary because some type (a) or (a') faces can be degenerated, but we cannot easily weaken this as other gluings inside $Y_{1}$ must be preserved.

We could also wonder if some torsion Vassiliev invariant can be found by this method, when the map $\beta$ takes values in a torsion $\mathbb{Z}$-module. But there may also be torsion invariants that cannot be found by this method. (This is suggested by the fact that the rationality results we can obtain in degree 2 by this method are not the best ones: see Appendix A.3). But we won't be interested in this problem here.

\section{A.2 Universality of $Z_{n}$ in the case of a free module}

Now we are going to see that if the map $\beta$ takes values in a free $\mathbb{Z}$-module or in a $\mathbb{R}$-vector space, then the corresponding $\operatorname{deg} \xi$ if it exists, or the corresponding integral, comes from $Z_{n}$. Thus, the only interest in choosing such other maps $\beta$ is to obtain better rationality results than those of Theorem 1.11.

Proposition A.1 If a map $\beta$ from the set of principal elements of $D_{n, k}(M)$ to some $\mathbb{R}$-vector space $\mathcal{E}$ obeys the $S T U$ ' relation, then the corresponding expression of $\operatorname{deg} \Psi$ is of the form $\mathcal{L}\left(Z_{n}(K)\right)$ where $\mathcal{L}$ is a linear map from $\mathcal{A}_{n}(M)$ to $\mathcal{E}$ which only depends on $\beta$.

Let $\mathcal{G}$ be the set of diagrams $\Gamma \in \mathcal{D}_{n}(M)$ which are principal and verify $u_{\Gamma} \geq$ $k-1$. 
We shall first define a map $\psi$ from $\mathcal{G}$ to $\mathcal{E}$. Then, we shall prove that $\psi$ extends to a linear map $\mathcal{L}$ defined on $\mathcal{A}_{n}(M)$, and that this $\mathcal{L}$ is the solution to our problem.

Let us first define $\psi$ from $\mathcal{G}$ to $\mathcal{E}$ by:

$$
\forall \Gamma, \quad \psi(\Gamma)=|\Gamma| \sum_{\substack{\Gamma^{\prime} \in D_{n, k}(M) \\ \Gamma^{\prime} \sim \Gamma}} \beta\left(\Gamma^{\prime}\right) .
$$

The existence of a linear extension of $\psi$ on $\mathcal{A}_{n}(M)$ will be deduced from the following facts:

(1) Any STU' relation implies the corresponding STU relation for $\psi$.

(2) This map $\psi$ is well-defined on chord diagrams and obeys the four-terms relation.

(3) The STU relation obtained from (1) allows us to express any diagram in $\mathcal{G}$ as a linear combination of chord diagrams.

Indeed, from (2) we deduce that $\psi$ defines a linear map from the space of degree $n$ chord diagrams with support $M$ modulo the four-terms relation. But we know that this space is identified with $\mathcal{A}_{n}(M)$ [BN1], so $\psi$ defines a linear map $\mathcal{L}$ on $\mathcal{A}_{n}(M)$. This map coincides with $\psi$ on $\mathcal{G}$, thanks to (1) and (3).

Now, to prove the identity

$$
\sum_{\Gamma \in \mathcal{G}} \beta(\Gamma) I_{L}(\Gamma)=\sum_{\Gamma \in \mathcal{D}_{n}(M)} \frac{I_{L}(\Gamma)}{|\Gamma|} \mathcal{L}([\Gamma])
$$

we have to check that if a diagram $\Gamma \in \mathcal{D}_{n}(M)$ is not represented in $\mathcal{G}$, then $I_{L}(\Gamma)=0$ or $\mathcal{L}([\Gamma])=0$. Suppose that $I_{L}(\Gamma) \neq 0$. Then $\Gamma$ is principal, hence $u_{\Gamma}<k-1$. Thus, $\Gamma$ can be expressed modulo STU as a linear combination of diagrams which have $k-1$ univalent vertices and are principal. But since $\mathcal{L}$ vanishes on them, it also vanishes on $\Gamma$.

Now let us prove the claims (1), (2) and (3).

Proof of (1) Consider a STU' relation; let $\Gamma$ be any diagram in this relation, and let $A$ be the pair of vertices involved.

Note that this STU' relation is defined by the labelled diagram $\Gamma / A$. We shall consider the sum of this relation over the set of relabellings of $\Gamma / A$, in other words, the set of the $(\Upsilon, \lambda)$, where $\Upsilon$ is a diagram isomorphic to $\Gamma / A$, and $\lambda$ is an isomorphism from $\Gamma / A$ to $\Upsilon$. 
This sum of relations, when applied to one of the three parts of the relation (the one of $\Gamma$ ), is a sum over the set of relabellings of $\Gamma$, that is, the set of pairs $\left(\Gamma^{\prime}, \lambda\right)$ where $\lambda$ is an isomorphism from $\Gamma$ to $\Gamma^{\prime}$. This set is in one-to-one correspondence with the product of the set of automorphisms of $\Gamma$ with the set of diagrams which are isomorphic to $\Gamma$. So, this sum gives precisely the corresponding term $\psi(\Gamma)$ of the STU relation.

This proves (1).

Proofs of (2) and (3) Since $k \leq 2 n, \psi$ is well-defined on the chord diagrams, and also on the diagrams with only one trivalent vertex. Then the four-terms relation is a consequence of the STU relation deduced from (1).

The proof of (3) is easy by induction: we just have to note that when we use a STU relation to express a diagram in terms of two diagrams with one more univalent vertex, then these two diagrams are principal provided that the first one is.

This can be generalised to the case when $\beta$ takes values in any free $\mathbb{Z}$-module $\mathcal{M}$, for $\mathcal{M}$ is canonically imbedded in the real vector space $\mathcal{M} \otimes \mathbb{R}$.

\section{A.3 Rationality results in degree 2}

In this last part of the Appendix, we shall restrict ourselves to the case when $L$ is a knot $K$.

Theorem 1.11 gives the same rationality result in degree 2 as the one of [Le], that is, a denominator of 48 . But the actual denominator is 24 , according to the formula

$$
Z_{2}=\frac{I_{K}(\theta)^{2}}{8}[\theta]^{2}+\left(v_{2}-\frac{1}{24}\right)[\bigotimes]
$$

where $v_{2} \in \mathbb{Z}$ is the degree 2 Vassiliev invariant which vanishes on the unknot $O$ and takes the value 1 on the trefoils. This formula comes from the Vassiliev universal property of $Z$ (Section 8), and the computation of

$$
I_{O}(\bigotimes)=\frac{1}{8}
$$

first made in [GMM] (not very difficult).

This actual denominator can be obtained in terms of degree theory as Polyak and Viro did [PV]. Let us recall their remarks, and specify the general context for which this improvement in degree two is a particular case.

Algebraic $8 \mathcal{G}$ Geometric Topology, Volume 2 (2002) 
Keep $N=3$, but consider the map $\beta$ from $D_{2,3}$ to $\mathbb{Z}$ defined by:

$$
\beta(\bigotimes)=0, \quad \beta(\bigotimes)=\frac{1}{24}
$$

independently of the labelling, and

$$
\beta(\bigotimes)=0 \text { or }-\frac{1}{24}
$$

according to the following rule: the labellings of this diagram are divided into two classes according to the parity of the number of edges oriented towards the trivalent vertex. Then give the value $-\frac{1}{24}$ to a class and 0 to the other. It is easy to see that the gluing conditions are realised for this map $\beta$. Therefore, this induces a degree

$$
\operatorname{deg} \Psi=v_{2}-\frac{1}{24} .
$$

The expression of $Z_{2}$ suggests that, to find better denominators, it would be preferable to manage to cancel the $-\frac{1}{24}$ term. This will be done by considering the expression $\frac{Z(K)}{Z(O)}$.

Proposition A.2 The expression

$$
\frac{Z(K)}{Z(O)}
$$

is equal to the expression defined the same way as $Z$, with a long knot: instead of an imbedding of $S^{1}$ into $\mathbb{R}^{3}$, we put the knot in the form of an imbedding of $\mathbb{R}$ into $\mathbb{R}^{3}$ which coincides with the inclusion of a given straight line of $\mathbb{R}^{3}$ outside some compact set.

We shall not prove this result here as it can be directly deduced from our next article $[\mathrm{P} 2]$ on the extension of the configuration space integral to tangles.

We are only going to show that the result of the integral on this new shape of knots is an invariant, while the cyclic order on the univalent vertices of the labelled diagrams is replaced with a total order.

This is expressed in the following lemma:

Lemma A.3 The same conditions as those of Subsection A.1 but in the case of a long knot, imply that $\Psi$ admits a degree. 
Proof Let us first define the compactification of the configuration spaces we use here. We imbed each $C_{\Gamma}$ into the compact space

$$
\mathcal{H}(G) \times[-\infty,+\infty]^{U} .
$$

This compactification has the same general properties as in the case of compact knots, except that it has new faces which correspond to the $\left(f, f^{\prime}\right) \in \overline{C_{\Gamma}}$ such that $f^{\prime}(U)$ meets $\pm \infty$.

Thanks to Remark 3.2, we can suppose that $\Gamma$ is connected. Now, the fact that $f^{\prime}(U)$ meets $\pm \infty$ implies that $f_{V}$ maps all univalent vertices to the straight line $l_{z}$. We conclude that this face is degenerated by means of the argument of Subsection 3.2.

Now, let us glue the anomalous faces. Note that the tangent map $\mathrm{d} K$ : $[-\infty,+\infty] \longrightarrow S^{2}$ verifies $\mathrm{d} K(-\infty)=\mathrm{d} K(+\infty)$, so defines a map from $S^{1}$ to $S^{2}$, which is the boundary of some map $\varphi: D^{2} \longrightarrow S^{2}$. It still has the property that $I_{L}(\theta)+2 I$ is an integer (Lemma 6.15). (The only difference is that this integer is now even, whereas it was odd in the case of an ordinary knot.)

With this new problem, if we choose a map $\beta$ which only depends on the diagram obtained by closing the total order of $U$ into a cyclic order, then this gives exactly the same rationality result as the one we would obtain with this map from the diagrams with a cyclic order, for the compact knot. So, we can obtain a better rationality result for $\frac{Z(K)}{Z(O)}$ than for $Z(K)$, only by looking for maps $\beta$ which do depend on the cut of the cyclic order.

In particular for $n=2$ and $N=3$, take the map $\beta$ which only depends on the orientations of the edges, such that

$$
\beta\left(ぬ \frac{1}{6}, \quad \beta(\ /)=-\frac{1}{6}\right.
$$

and which vanishes on any other diagram. It naturally extends to generalised diagrams and satisfies all gluings. The corresponding expression $\operatorname{deg} \Psi$ is equal to $v_{2}$.

(Actually, no better result can be obtained with another map $\beta$ when $N=3$ ). 


\section{References}

[AF $]$ D. Altschuler and L. Freidel, Vassiliev knot invariants and Chern-Simons perturbation theory to all orders, arXiv:q-alg/9603010, to appear in Comm. Math. Phys.

[BN1] D. Bar-Natan, On the Vassiliev knot invariants, Topology 34 (1995), 423-472.

[BN2] D. Bar-Natan, Perturbative Chern-Simons Theory, Journal of Knot Theory and its Ramifications 4-4 (1995) 503-548.

[BS] D. Bar-Natan, A. Stoimenow, The Fundamental Theorem of Vassiliev Invariants, Geometry and Physics, lecture notes in pure and applied mathematics 184, 101-134, arXiv:q-alg/9702009

[BT] R.Bott and C.Taubes, On the self-linking of knots, Jour. Math. Phys. 35 (10) (1994), 5247-5287.

[GMM] E. Guadagnini, M. Martellini \& M. Mintchev, Chern-Simons field theory and link invariants, Nucl.Phys B330 (1990) 575-607.

[Ko] M. Kontsevich, Feynman diagrams and low-dimensional topology, First European Congress of Mathematics II 97-121, Birkhäuser Basel 1994.

[Le] T. Q. T. Le, On denominators of the Kontsevich integral and the universal perturbative invariant of 3-manifolds, q-alg/9704017 preprint, April 1997.

[LM] T. Q. T. LE and Jun MuraKami, The universal Vassiliev-Kontsevich invariant for framed oriented links, Compositio Mathematica 102 (1996), 41-64.

[Na] R.Narasimhan, Analysis on real and complex manifolds, Advanced study in Pure Mathematics 1 (1968).

[P1] S. Poirier, Rationality Results for the Configuration Space Integral of Knots, arXiv:math. GT/9901028

[P2] S. Poirier, The limit configuration space integral for tangles and the Kontsevich integral, arXiv:math.GT/9902058

[PV] M. Polyak and O. Viro, On the Casson knot invariant, Uppsala University preprint, March 1996.

[Th] Dylan P. Thurston, Integral Expressions for the Vassiliev Knot Invariants, arXiv:math.QA/9901110

[Ya] Su-Win Yang, Feynman Integral, Knot Invariant and Degree Theory of maps, arXiv:q-alg/9709029

For more references on Vassiliev invariants, see Bar-Natan's bibliography: http://www.ma.huji.ac.il/ drorbn/VasBib/VasBib.html

3 bis rue Auguste Dollfus, 76600 Le Havre, France

Email: spoirier@altern.org

Received: 30 November 2002 Revised: 15 July 2002 